\title{
Conductance fluctuations in chaotic systems with tunnel barriers
}

\author{
Daniel Waltner, ${ }^{1}$ Jack Kuipers, ${ }^{1}$ Philippe Jacquod, ${ }^{2,3,4}$ and Klaus Richter ${ }^{1}$ \\ ${ }^{1}$ Institut für Theoretische Physik, Universität Regensburg, D-93040 Regensburg, Germany \\ ${ }^{2}$ Physics Department, University of Arizona, Tucson, Arizona 85721, USA \\ ${ }^{3}$ College of Optical Sciences, University of Arizona, Tucson, Arizona 85721, USA \\ ${ }^{4}$ Département de Physique Théorique, Université de Genève, CH-1211 Genève
}

(Received 23 August 2011; revised manuscript received 24 November 2011; published 12 January 2012)

\begin{abstract}
Quantum effects are expected to disappear in the short-wavelength, semiclassical limit. As a matter of fact, recent investigations of transport through quantum chaotic systems have demonstrated the exponential suppression of the weak localization corrections to the conductance and of the Fano factor for shot noise when the Ehrenfest time $\tau_{E}$ exceeds the electronic dwell time $\tau_{D}$. On the other hand, conductance fluctuations, an effect of quantum coherence, retain their universal value in the limit $\tau_{E} / \tau_{D} \rightarrow \infty$, when the system is ideally coupled to external leads. Motivated by this intriguing result we investigate conductance fluctuations through quantum chaotic cavities coupled to external leads via (tunnel) barriers of arbitrary transparency $\Gamma$. Using the trajectory-based semiclassical theory of transport, we find that the linear $\tau_{E}$ dependence of the conductance variance shows a nonmonotonous, sinusoidal behavior as a function of $\Gamma$. Most notably, we find an increase of the conductance fluctuations with $\tau_{E}$, above their universal value, for $\Gamma \lesssim 0.5$. These results, confirmed by numerical simulations, show that, contrary to common wisdom, effects of quantum coherence may increase in the semiclassical limit, under special circumstances.
\end{abstract}

DOI: 10.1103/PhysRevB.85.024302

PACS number(s): 03.65.Sq, 05.45.Mt, 73.23.Ad

\section{INTRODUCTION}

Since the foundation of quantum physics there has been huge interest in the nontrivial transition from the quantum to the classical regime. An important observation in this context is the Ehrenfest theorem stating that the dynamics of quantum-mechanical expectation values is determined by the classical equations of motion. ${ }^{1}$ Going beyond expectation values, an Ehrenfest time scale has been identified as the time below which the quantum time evolution is well approximated by the corresponding classical dynamics. ${ }^{2}$ The Ehrenfest time is the time it takes for the chaotic classical dynamics to stretch an initially narrow wave packet to some relevant classical length scale such as the system size $L$. Since the stretching is exponential in classically chaotic systems, one has

$$
\tau_{E}=\frac{1}{\lambda} \ln \frac{p_{F} L}{\hbar},
$$

with the Lyapunov exponent $\lambda$ of the classical dynamics, the initial spread $\hbar / p_{F}$ of the wave packet, and the Fermi momentum $p_{F}$.

In recent years, there has been much interest in determining the influence of the Ehrenfest time on stationary transport quantities such as the conductance, ${ }^{3-8}$ its variance, ${ }^{6,9}$ and its behavior under decoherence, ${ }^{3,10-12}$ shot noise, ${ }^{13-15}$ and higher moments of the current, ${ }^{16,17}$ and on time dependent quantities such as the spectral form factor, ${ }^{8,18-20}$ the survival probability, ${ }^{21,22}$ and the fidelity. ${ }^{23}$ Most of these papers used the trajectory-based semiclassical approach to transport, ${ }^{24-26}$ which currently is the method of choice for investigating Ehrenfest-time dependences of quantum observables. ${ }^{6,27}$ The leading-order quantum correction to the conductance was found in Refs. 3 and 5-7 to decay exponentially with the Ehrenfest time. Qualitatively speaking this can be understood by noting first that this contribution originates from loop diagrams [see Fig. 3(b)], and as such depends on the return probability, and second that, neglecting system-dependent nongeneric processes, this return probability contribution essentially vanishes for times shorter than the Ehrenfest time. Such an intuitive interpretation of the Ehrenfest-time dependence does not always work: though inherently of nonclassical nature, the leading-order contribution to the conductance variance of systems ideally coupled to external leads turns out to be independent of the Ehrenfest time. ${ }^{6,9}$

In this paper, we determine semiclassically the Ehrenfesttime dependence of the variance, $\operatorname{var} G(E)$, of the conductance $G(E)$ for a chaotic system coupled to external leads via nonideal contacts modeled by tunnel barriers of transparency $\Gamma \leqslant 1$. The situation is depicted in Fig. 1. The presence of tunnel barriers has the dramatic effect that var $G(E)$ increases or decreases with $\tau_{E}$, depending on the value of $\Gamma$. For $\Gamma \lesssim 0.5$, we even observe an enhancement of the variance above the universal value in the presence of time-reversal symmetry for equal lead widths of $\operatorname{var}(G)^{\mathrm{RMT}}=\left[1+(1-\Gamma)^{2}\right] / 8,{ }^{28}$ upon increasing $\tau_{E}$. This is very surprising, given the quantal nature of the conductance fluctuations. In the range $0.5 \lesssim \Gamma<1$ we find a reduction of $\operatorname{var} G(E)$ as $\tau_{E}$ increases that is strongest around $\Gamma \simeq 0.8$, and recover the $\tau_{E}$-independent behavior of $\operatorname{var} G(E)$ at $\Gamma=1$ observed in Refs. 6 and 9. The precise dependence on $\Gamma$ is depicted in Fig. 2.

To obtain these results we first, in Sec. II, introduce the semiclassical approximation to the conductance variance. Then, in Sec. III, we analytically calculate the $\Gamma$ dependence of the diagrammatic contributions to the variance, to leading order in the inverse total number of open channels and linear in the Ehrenfest time. We list all relevant diagrams and calculate their contributions. Given their number, we also identify the most relevant ones and specify the range in $\Gamma$ where they are particularly important. In Sec. IV we present numerical results that confirm our analytical results, and conclude in Sec. V. 


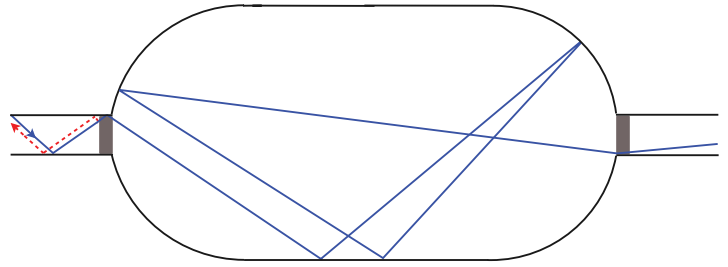

FIG. 1. (Color online) A chaotic cavity coupled to external leads via tunnel barriers of transparency $\Gamma \leqslant 1$ (gray boxes). A classical trajectory traversing the system is shown by a solid (blue) line. The second dashed (red) line on the left indicates a trajectory backreflected at the barrier. This occurs with probability $1-\Gamma$.

\section{SEMICLASSICAL APPROACH}

Within the scattering approach to quantum transport, ${ }^{29,30}$ the energy averaged conductance $G(E)$ (in units of $2 e^{2} / h$ ) can be expressed in terms of the transmission $\mathbf{t}$ as

$$
G(E)=\left\langle\operatorname{Tr}\left(\mathbf{t t}^{\dagger}\right)\right\rangle
$$

with $\langle\cdots\rangle$ denoting an average over an energy window that is classically small but quantum mechanically large. This leads to the following expression for the variance:

$$
\operatorname{var} G(E)=\left\langle\left[\operatorname{Tr}\left(\mathbf{t t}^{\dagger}\right)\right]^{2}\right\rangle-\left\langle\operatorname{Tr}\left(\mathbf{t t}^{\dagger}\right)\right\rangle^{2} .
$$

The scattering matrix elements are related by the Fisher-Lee relation $^{31}$ to the projection of the Green function onto the transverse directions in the leads. Performing the projection to leading order in $\hbar$ and approximating the Green function semiclassically, one obtains

$$
t_{a, b} \approx \frac{1}{\sqrt{T_{H}}} \sum_{\gamma(a \rightarrow b)} A_{\gamma} e^{(i / \hbar) S_{\gamma}},
$$

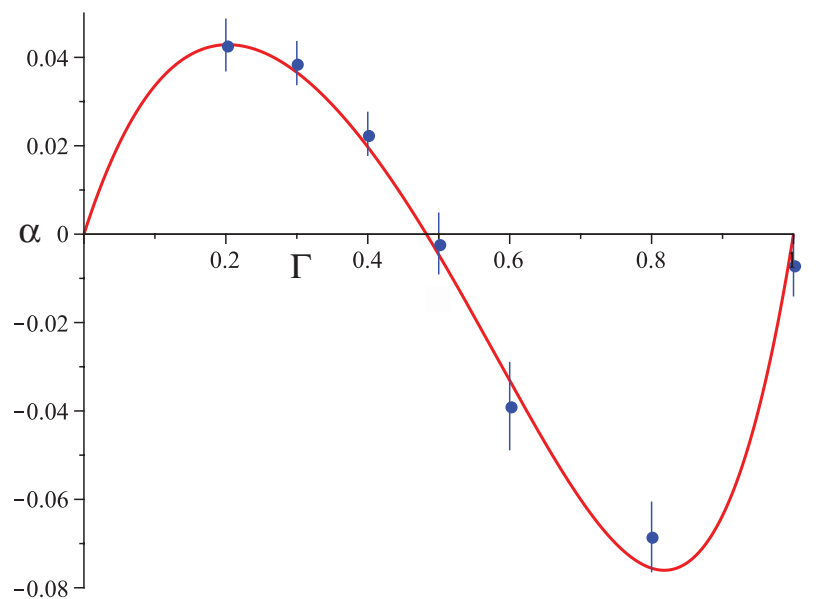

FIG. 2. (Color online) Dependence of the transmission fluctuations on cavity lead coupling $\Gamma$. The function $\alpha \tau_{E} / \tau_{D}$, defined in Eqs. (77) and (78), measures the deviation from the universal random matrix theory (RMT) variance. In this graph the parameter $\alpha$ is plotted as a function of the tunnel barrier transparency $\Gamma$ for time-reversal symmetric systems. The solid red line gives the analytical prediction, Eq. (78), and the blue dots are results of numerical simulations. Error bars indicate the standard deviation over the ensemble of calculated data. with the Heisenberg time $T_{H}$, the time conjugate to the mean level spacing. Here the sum is over the scattering trajectories $\gamma$, which connect channel $a$ in the entrance (or say left) lead and channel $b$ in the exit (or right) lead in Fig. 1. The summands contain rapidly oscillating phases depending on the classical actions $S_{\gamma}$ of the considered classical trajectories, and classical stability prefactors $A_{\gamma}$ whose precise form is given, for example, in Ref. 26.

Inserting Eq. (4) into Eq. (2) we obtain the semiclassical expression for the conductance,

$$
G(E) \approx\left\langle\frac{1}{T_{H}} \sum_{a, b} \sum_{\gamma, \gamma^{\prime}(a \rightarrow b)} A_{\gamma} A_{\gamma^{\prime}}^{*} e^{(i / \hbar)\left(S_{\gamma}-S_{\gamma^{\prime}}\right)}\right\rangle .
$$

Using Eq. (4) in Eq. (3) yields the semiclassical expression for its variance,

$$
\begin{aligned}
\operatorname{var} & G(E) \\
\approx & \left\langle\frac{1}{T_{H}^{2}} \sum_{\substack{a, b \\
c, d}} \sum_{\substack{\gamma, \gamma^{\prime}(a \rightarrow b) \\
\xi, \xi^{\prime}(c \rightarrow d)}} A_{\gamma} A_{\gamma^{\prime}}^{*} A_{\xi} A_{\xi^{\prime}}^{*} e^{(i / \hbar)\left(S_{\gamma}-S_{\gamma^{\prime}}+S_{\xi}-S_{\xi^{\prime}}\right)}\right\rangle \\
& -\left\langle\frac{1}{T_{H}} \sum_{a, b} \sum_{\gamma, \gamma^{\prime}(a \rightarrow b)} A_{\gamma} A_{\gamma^{\prime}}^{*} e^{(i / \hbar)\left(S_{\gamma}-S_{\gamma^{\prime}}\right)}\right\rangle^{2}
\end{aligned}
$$

with the channel sums in Eqs. (5) and (6) running over all open lead channels ( $N_{L}$ in the left and $N_{R}$ in the right lead). If we consider contributions in the first term in Eq. (6) where $\gamma$ and $\gamma^{\prime}$ form a correlated pair (with self-encounters) and $\xi$ and $\xi^{\prime}$ form a separate correlated pair, we simply recreate the second term. We can thus remove the second term in the above equation by removing such pairs from the semiclassical treatment of the first term. In terms of trajectories we then obtain

$$
\begin{aligned}
\operatorname{var} G(E) \approx & \left\langle\left\langle\frac{1}{T_{H}^{2}} \sum_{\substack{a, b \\
c, d}} \sum_{\substack{\gamma, \gamma^{\prime}(a \rightarrow b) \\
\xi, \xi^{\prime}(c \rightarrow d)}} A_{\gamma^{\prime} \rightarrow d} A_{\gamma^{\prime}}^{*} A_{\xi} A_{\xi^{\prime}}^{*}\right.\right. \\
& \left.\times e^{(i / \hbar)\left(S_{\gamma}-S_{\gamma^{\prime}}+S_{\xi}-S_{\xi^{\prime}}\right)}\right\rangle,
\end{aligned}
$$

where the trajectories $\gamma, \gamma^{\prime}$ go from channel $a$ in the entrance lead to channel $b$ in the exit lead. Likewise trajectories $\xi, \xi^{\prime}$ go from channel $c$ to channel $d$. Because we have removed terms from correlated trajectories where $\gamma \approx \gamma^{\prime}$ and $\xi \approx \xi^{\prime}$ [this restriction is denoted by the double bracket in Eq. (7)] we are left with quadruplets where all four trajectories interact through encounters.

Before performing the energy average the approximations for $G(E)$ and var $G(E)$ in Eqs. (5) and (7) are rapidly fluctuating as a function of energy for $\hbar \rightarrow 0$. Thus only contributions from very similar trajectories survive the average. The classical contribution to Eq. (5) results from equal trajectories $\gamma=\gamma^{\prime}$, the so-called diagonal approximation; ${ }^{24,26}$ for an illustration, see Fig. 3(a). Here Eq. (5) yields

$$
G(E)^{[3(a)]}=\frac{1}{T_{H}} \sum_{a, b} \sum_{\gamma(a \rightarrow b)}\left|A_{\gamma}\right|^{2} .
$$



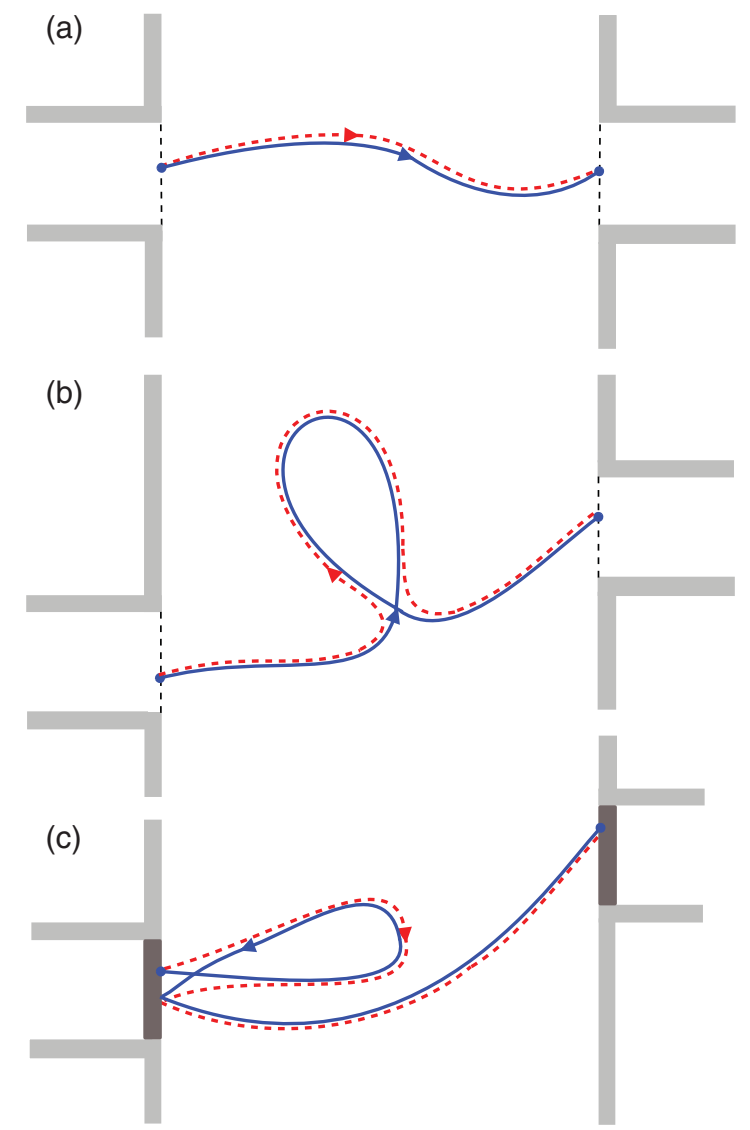

FIG. 3. (Color online) Schematic drawing of trajectory pairs contributing semiclassically to the energy averaged conductance. (a) A pair of identical trajectories leading to the classical contribution to the conductance. (b) The pair depicted here differs at a selfencounter and leads to the weak-localization quantum correction to the conductance. (c) An additional pair of orbits that needs to be considered in the presence of tunnel barriers. This diagram is obtained from (b) by shrinking the left link until the encounter touches the tunnel barrier. An analogous configuration can be formed by removing the right link from Fig. 3(b).

From here on, superscripts refer to the corresponding figure. To evaluate the remaining $\gamma$ summation, the sum rule for open systems ${ }^{25}$ that transforms the sum over orbits into an integral over their durations is applied,

$$
\sum_{\gamma}\left|A_{\gamma}\right|^{2} \approx \int_{0}^{\infty} d T e^{-T / \tau_{D}}
$$

Here $\tau_{D}$ is the dwell time of the cavity, i.e., the typical time a classical particle remains inside the chaotic system. This is given by $\tau_{D}=T_{H} / N$ with $N \equiv N_{L}+N_{R}$. This finally yields

$$
G(E)^{[3(a)]} \approx \frac{N_{L} N_{R}}{N_{L}+N_{R}} .
$$

Quantum corrections to this result are obtained from pairs of slightly different trajectories. The considered trajectories are almost identical differing only in how they are connected within self-encounters; for the pair considered in Ref. 25, see Fig. 3(b). Here the orbits possess close self-encounters with one orbit crossing and the other anticrossing there leading to a different direction of traversal of the closed loop. Considering

this pair in Eq. (5) leads to the leading-order quantum correction to the conductance. To determine its contribution the action difference between the partner trajectories and the number of crossings needs to be determined. The calculation is done here within the phase-space approach; in the context of the conductance it was first performed in Ref. 32. We will follow the latter approach throughout this paper. There a Poincaré surface of section is considered inside the encounter region and the difference along the stable and unstable directions of the piercing points of the two stretches, $s$ and $\boldsymbol{u}$, respectively, is used to characterize an encounter. In terms of these coordinates the action difference for the orbit pair in Fig. 3(b) is given by $\Delta S=s u{ }^{32}$ The weight $w(s, u)$ that additionally depends on the duration of the orbit $T$ measuring the number of encounters is obtained from the ergodicity of the flow as ${ }^{32}$

$$
w(s, u)=\frac{\left(T-2 t_{\mathrm{enc}}\right)^{2}}{2 \Omega t_{\mathrm{enc}}},
$$

where $t_{\mathrm{enc}} \equiv 1 / \lambda \ln \left(c^{2} /|s u|\right)$ is the duration of the encounter and $\Omega$ the volume of the energy shell of the corresponding closed system. The $c^{2}$ is a classical action of order unity that will later be related to the Ehrenfest time. In general, the action difference $\Delta S$ and the weight $w(\boldsymbol{s}, \boldsymbol{u})$ depend on the trajectory configuration considered. In total we obtain for the quantum correction $\delta G(E)$ resulting from the diagram in Fig. 3(b)

$$
\begin{aligned}
\delta G(E)^{[3(b)]} & \approx \frac{N_{L} N_{R}}{T_{H}} \int_{-c}^{c} d s d u \int_{2 t_{\mathrm{enc}}}^{\infty} d T w(s, u) e^{i \Delta S / \hbar} e^{-\left(T-t_{\mathrm{enc}}\right) / \tau_{D}} \\
& \approx-\frac{N_{L} N_{R}}{\left(N_{L}+N_{R}\right)^{2}} e^{-\tau_{E} / \tau_{D}}
\end{aligned}
$$

with $\tau_{E} \equiv 1 / \lambda \ln \left(c^{2} / \hbar\right)$. In the first line additionally the survival probability correction during the encounter ${ }^{32}$ is taken into account. During the encounter the stretches are so close that the orbit escapes either during the first stretch or does not escape at all leading to the enhanced survival probability in Eq. (12). The $s, u$ integrals in Eq. (12) are performed as described in Refs. 6 and 8, by substituting $s u=c^{2} x$ and $\sigma=c / u$. The $\sigma$ integral then essentially cancels the $t_{\text {enc, }}$ in the denominator and the $x$ integral yields (after a partial integration) the contribution $-1 /\left(\tau_{D} T_{H}\right) e^{-\tau_{E} / \tau_{D}}$.

To treat orbits differing at several places, Ref. 32 introduces the splitting of the orbit into encounters and links. During the encounters the orbits are close to themselves but the orbit and its partner are differently connected. Due to the exponential separation of neighboring trajectories in the chaotic case these last essentially an Ehrenfest time, as will become clear from the calculation below. The stretches are connected by the links, where "links" denote the long parts of the trajectory where the trajectory and its partner are essentially identical (up to time-reversal symmetry). With a suitable change of variables in the calculation, in the RMT limit $\tau_{E} \rightarrow 0$ one can treat different encounters as distinct, and separate the semiclassical contribution into a product of contributions over each of the links and encounters. The total contribution can therefore be obtained by diagrammatic rules. ${ }^{16,32}$ Away from this limit, and for the Ehrenfest-time dependence, the encounters may start to overlap and for the conductance variance the trajectories can be seen to meet and surround periodic orbits trapped inside 
the system, i.e., they have encounters with periodic orbits, and must be treated as part of a continuous family. ${ }^{6}$

When we include tunnel barriers (as in Fig. 1), three main changes occur that were originally described in Ref. 33:

(i) The particles enter and leave the cavity with the probability $\Gamma$, which leads to a factor $\Gamma^{2}$ for each trajectory pair.

(ii) While without tunnel barriers every particle that hits the lead leaves the system, now only the ratio $\Gamma$ of the particles hitting the lead leaves the cavity. For the links, the effective dwell time is therefore $\tau_{D} / \Gamma$ and the dwell time in the exponential in Eq. (9) should be replaced by this effective dwell time. However, if trajectory stretches are correlated, as they are during encounters, then the whole configuration is lost if just one stretch of the encounter leaves the system. This happens with a probability $p_{n} \equiv 1-(1-\Gamma)^{n}$ for $n$ correlated stretches. The dwell time in such a situation is therefore replaced by $\tau_{D} /\left[1-(1-\Gamma)^{n}\right]$.

(iii) Additional encounter diagrams become possible; for an example which contributes to the energy averaged conductance $G(E)$, see Fig. 3(c). In this case one encounter stretch can be moved into the lead forcing the other to be backreflected at the opening. Note that configurations where both stretches are backreflected at the opening are already taken into account by the modified dwell time explained above.

Although the effective dwell times are altered by the tunnel barriers, the action difference and weight functions are unaffected so that in the RMT limit $\left(\tau_{E} \rightarrow 0\right)$ contributions can still be obtained by diagrammatic rules: The contribution of each link is now given by $(\Gamma N)^{-1}$. The stretches of an encounter of $n$ orbits yield $-p_{n} N$. For the Ehrenfest-time dependence, however, these changes render the calculation of the contributions to the conductance variance much more difficult compared to $\Gamma=1$ : Due to the discontinuous form of the effective dwell time, the contributions from diagrams with a different number of surroundings of trapped periodic orbits need to be split and treated separately. Also the possibility for encounter stretches to be backreflected at the tunnel barriers increases the number of diagrams considerably.

\section{DIAGRAMMATIC CONTRIBUTIONS}

Here we calculate the leading order in $1 / N$ contributions to the variance of the conductance for nonzero Ehrenfest time in the presence of tunnel barriers. We show all the relevant diagrams and calculate their contributions. The results given here are valid in the unitary case; results for the orthogonal case can be obtained by multiplying the total by a factor of 2 .

\section{A. Discrete encounters}

We start with the contributions important in the RMT limit, which allow us to recover the RMT result. The corresponding RMT calculation was performed in Ref. 28 by Brouwer and Beenakker. First we consider two two-encounters (encounters involving two trajectory stretches) in a row, see Fig. 4(a). This diagram also occurred in the $\Gamma=1$ treatment of Ref. 6 , but we will explain how for $\Gamma \neq 1$ other diagrams with backreflected stretches can be derived from this one.

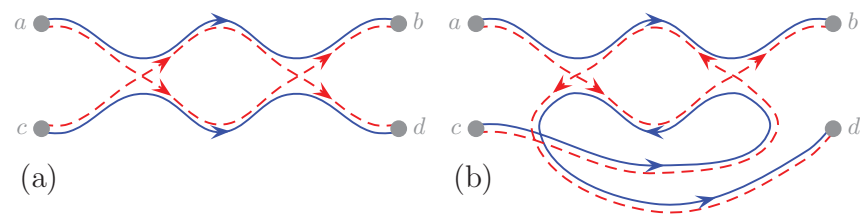

FIG. 4. (Color online) (a) A diagram possessing two twoencounters in a row. This diagram does not require time-reversal symmetry unlike the corresponding diagram in (b).

When the encounters are inside the cavity and not touching the tunnel barriers, as depicted in Fig. 4(a), we start with Eq. (7) and use the sum rule (9), the action difference $\Delta S(\boldsymbol{s}, \boldsymbol{u})=s_{1} u_{1}+s_{2} u_{2}$ (where the subscripts refer to the different encounters), and the weight function, ${ }^{32}$

$$
w(\boldsymbol{s}, \boldsymbol{u})=\frac{\left(T_{1}-t_{\mathrm{enc}, 1}-t_{\mathrm{enc}, 2}\right)^{2}\left(T_{2}-t_{\mathrm{enc}, 1}-t_{\mathrm{enc}, 2}\right)^{2}}{4 \Omega^{2} t_{\mathrm{enc}, 1} t_{\mathrm{enc}, 2}},
$$

containing the durations of the two trajectories indicated by solid lines in Fig. 4(a), $T_{1}$ and $T_{2}$, respectively, and the durations of the two encounters of the trajectories, ${ }^{34}$

$$
t_{\text {enc }, i} \equiv \frac{1}{\lambda} \ln \frac{c^{2}}{\left|s_{i} u_{i}\right|}, \quad i \in\{1,2\},
$$

with a classical constant $c$ again of the order 1. From Eqs. (6) and (9) we write

$$
\begin{aligned}
\operatorname{var} G^{[4]}= & \frac{\Gamma^{4} N_{L}^{2} N_{R}^{2}}{T_{H}^{2}} \prod_{i=1}^{6} \int_{0}^{\infty} d t_{i} e^{-\Gamma t_{i} / \tau_{D}} \int_{-c}^{c} d \boldsymbol{s} d \boldsymbol{u} \frac{1}{\Omega^{2}} \\
& \times \frac{e^{(i / \hbar) s u}}{t_{\mathrm{enc}, 1} t_{\mathrm{enc}, 2}} e^{-p_{2}\left(t_{\mathrm{enc}, 1}+t_{\mathrm{enc}, 2}\right) / \tau_{D}},
\end{aligned}
$$

with the superscript again referring to the corresponding figure, Fig. 4. As explained in Sec. II, the trajectory quadruplet leads to the overall factor $\Gamma^{4}$ while the sum over possible channels provides $N_{L}^{2} N_{R}^{2}$. The six links have an effective dwell time of $\tau_{D} / \Gamma$, while each two-encounter experiences the dwell time $\tau_{D} / p_{2}$ as explained at the end of the last section. The $s, u$ integrals are performed, as described after Eq. (12), by substituting $s_{i} u_{i}=c^{2} x_{i}$ and $\sigma_{i}=c / u_{i}$. Each $x_{i}$ integral yields (after a partial integration) the contribution $-p_{2} /\left(\tau_{D} T_{H}\right) e^{-p_{2} \tau_{E} / \tau_{D}}$ as already obtained for the conductance for $\Gamma=1$. Finally the $t_{i}$ integrals yield

$$
\operatorname{var} G^{[4]}=\frac{N_{L}^{2} N_{R}^{2}}{\left(N_{L}+N_{R}\right)^{4}} \frac{p_{2}^{2}}{\Gamma^{2}} e^{-2 p_{2} \tau_{E} / \tau_{D}},
$$

which generalizes the result for $\Gamma=1$ from Ref. 6. The representation of Eq. (15) and the integrals to arrive at Eq. (16) also nicely illustrate how the diagrammatic rules introduced above arise in this context.

We now turn to the new diagrams that arise due to the tunnel barriers where some of the links are shrunk until an encounter touches a barrier. First we consider the case in which just one link connecting the encounter to the opening is removed and the corresponding encounter stretch now starts in the opening, as in the example in Fig. 3(c). As the stretches during an encounter lie very close to each other, the other encounter stretch has to be backreflected at the opening (so only one link is lost). This contribution can therefore only 
exist for $\Gamma \neq 1$. The changes in the analytical calculation mainly affect the weight function; see also Refs. 8, 21, 22, and 33. If we shrink a link on trajectory 1 then, compared to Eq. (13), the orbit of duration $T_{1}$ now only involves two links so the factor $\left(T_{1}-t_{\mathrm{enc}, 1}-t_{\mathrm{enc}, 2}\right)^{2} / 2$ is replaced by $\left(T_{1}-t_{\mathrm{enc}, 1}-t_{\mathrm{enc}, 2}\right)$. Moreover, for the encounter that touches the barrier, $t_{\mathrm{enc}, i}$ is replaced by an integration variable $t^{\prime}$ that is integrated from zero to the $t_{\mathrm{enc}, i}$ defined in Eq. (14). This variable measures the length of the encounter that remains inside the system, i.e., which has not yet been moved into the lead. Performing again the steps described after Eq. (14) yields an expression similar to Eq. (15) but with five instead of six link factors and $t_{\mathrm{enc}, i}$ in the exponential in the second line replaced by an integration variable $t^{\prime}$ that is integrated from zero to $t_{\mathrm{enc}, i}$. Because half of the encounter is backreflected at the tunnel barrier, additionally this contribution is multiplied by $(1-\Gamma)$. The contribution from the lower limit of the $t^{\prime}$ integral is zero, ${ }^{8,17}$ leading to

$$
\operatorname{var} G^{[4-1 l]}=-\frac{4 N_{L}^{2} N_{R}^{2}}{\left(N_{L}+N_{R}\right)^{4}} \frac{(1-\Gamma) p_{2}}{\Gamma} e^{-2 p_{2} \tau_{E} / \tau_{D}},
$$

with the " $-1 l$ " denoting that one link was removed. The prefactor 4 is due to the fact that there are four such links we can remove. Analogously, the contribution where two links that connect two different encounters to the opening are removed is

$$
\operatorname{var} G^{[4-2 l]}=\frac{4 N_{L}^{2} N_{R}^{2}}{\left(N_{L}+N_{R}\right)^{4}}(1-\Gamma)^{2} e^{-2 p_{2} \tau_{E} / \tau_{D}},
$$

where there are again four possibilities of picking two links to remove.

Additionally we can, and this is a possibility which also exists for $\Gamma=1$, remove both links connecting the same encounter to the opening. This means that the trajectories tunnel straight into the encounter. As the encountering orbits are so close together this means that $a=c$ or $b=d$ so that there is only one channel summation in the lead where the encounter touches. Also we have two links fewer and one integral over the part of the encounter that remains inside the system, yielding

$$
\operatorname{var} G^{[4-2 l(s)]}=-\frac{\left(N_{L}^{2} N_{R}+N_{L} N_{R}^{2}\right)}{\left(N_{L}+N_{R}\right)^{3}} p_{2} e^{-2 p_{2} \tau_{E} / \tau_{D}},
$$

where the additional "(s)" in the superscript indicates that two links were removed at the same encounter. We can further remove one link from the other encounter to obtain

$$
\operatorname{var} G^{[4-3 l]}=\frac{2\left(N_{L}^{2} N_{R}+N_{L} N_{R}^{2}\right)}{\left(N_{L}+N_{R}\right)^{3}} \Gamma(1-\Gamma) e^{-2 p_{2} \tau_{E} / \tau_{D}} .
$$

Finally, when all four links connecting the encounter to the leads are removed, we have

$$
\operatorname{var} G^{[4-41]}=\frac{N_{L} N_{R}}{\left(N_{L}+N_{R}\right)^{2}} \Gamma^{2} e^{-2 p_{2} \tau_{E} / \tau_{D}} .
$$

With time-reversal symmetry, however, we can also have Fig. 4(b) where, because channels $a$ and $c$ are in the left lead and channels $b$ and $d$ in the right lead, we cannot shrink more than two links simultaneously. Similarly, we cannot remove two links from the same encounter. Since $p_{2}=2 \Gamma-\Gamma^{2}$, the contributions Eqs. (19)-(21) actually cancel so that the

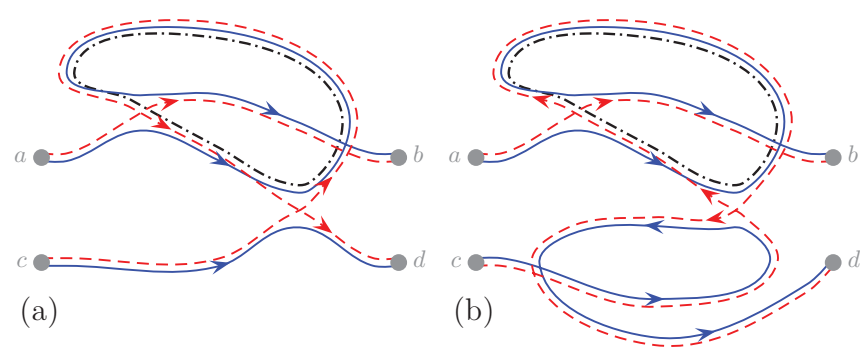

FIG. 5. (Color online) (a) A diagram possessing two independent two-encounters with a periodic orbit (dashed-dotted line). While this diagram does not require time-reversal symmetry, the corresponding diagram (b) does.

diagram in Fig. 4(b) and the ones obtained by cutting links provide the same contribution as the diagram in Fig. 4(a). Time-reversal symmetry then still gives a factor 2 in this case, while for all the following cases, diagrams related by time-reversal symmetry provide the same contributions directly.

Next we treat the diagrams with two two-encounters that lie along a trapped periodic orbit, depicted in Fig. 5. Although the links connect, the encounter stretches in a different way compared to Fig. 4; these diagrams again contain two twoencounters and the same number of links. The possibilities for shrinking links are identical as for Fig. 4(b) and so each diagram provides the same contributions as above and the same total contribution as the configuration in Fig. 4(a). They are multiplied, however, by a factor 2 since the orbits here have two possibilities to traverse the enclosed periodic orbits (schematically we can also reflect the diagrams horizontally).

The last relevant diagram type to obtain the RMT result is shown in Fig. 6. This diagram contains one three-encounter with a periodic orbit. Its contribution is calculated in an analogous way to Eq. (15). Here we have one link fewer, one encounter instead of two, and involving three rather than two orbit stretches. Therefore we have

$$
\operatorname{var} G^{[6]}=-\frac{2 N_{L}^{2} N_{R}^{2}}{\left(N_{L}+N_{R}\right)^{4}} \frac{p_{3}}{\Gamma} e^{-p_{3} \tau_{E} / \tau_{D}} .
$$

Alternatively this result can be obtained from the contribution of a three-encounter, $K_{1}$, in the Appendix of Ref. 8 by including the correct dwell times as well as the contributions from the links.

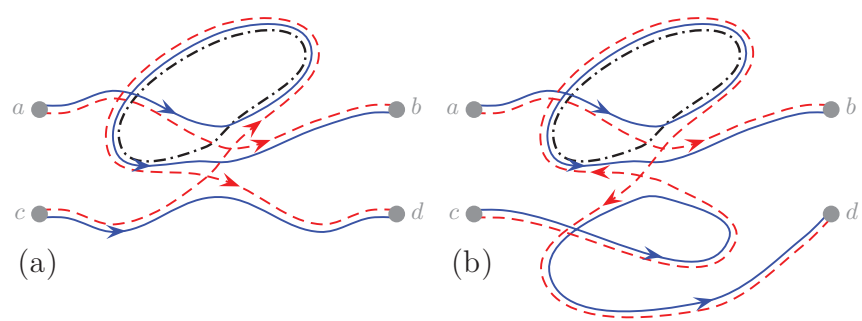

FIG. 6. (Color online) (a) A diagram with one three-encounter with a periodic orbit (dashed-dotted line). Diagram (a) does not require time-reversal symmetry while diagram (b) does. 
Also in this case encounter stretches can be shrunk and removed: First removing one link connecting the encounter to the opening yields

$$
\operatorname{var} G^{[6-1 l]}=\frac{8 N_{L}^{2} N_{R}^{2}}{\left(N_{L}+N_{R}\right)^{4}}(1-\Gamma)^{2} e^{-p_{3} \tau_{E} / \tau_{D}}
$$

and second removing both links,

$$
\operatorname{var} G^{[6-2 l]}=\frac{2\left(N_{L} N_{R}^{2}+N_{L}^{2} N_{R}\right)}{\left(N_{L}+N_{R}\right)^{3}} \Gamma(1-\Gamma) e^{-p_{3} \tau_{E} / \tau_{D}} .
$$

Having calculated all the contributions in the RMT limit, we can obtain the RMT result by setting $\tau_{E}=0$. When summing the the results in Eqs. (16)-(24), we obtain

$$
\begin{aligned}
\operatorname{var} G^{\mathrm{RMT}}= & \frac{N_{L} N_{R} \Gamma^{6}}{\left(N_{L}+N_{R}\right)^{6}}\left[N_{L}^{2} N_{R}^{2}\left(4-8 \Gamma+6 \Gamma^{2}\right)\right. \\
& +\left(N_{L}^{3} N_{R}+N_{L} N_{R}^{3}\right)\left(2-2 \Gamma+\Gamma^{2}\right) \\
& \left.+\left(N_{R}^{4}+N_{L}^{4}\right)\left(2 \Gamma-2 \Gamma^{2}\right)\right]
\end{aligned}
$$

which agrees with the RMT prediction in Ref. 28. In Appendix B, we use these diagrams to obtain the RMT result when each channel has a different tunneling probability and extend this treatment to shot noise in Appendix C. In the next subsection we turn to nonzero Ehrenfest time.

\section{B. Periodic orbit encounters}

Having gone through all the diagrams that contribute at zero Ehrenfest time, we now turn to those diagrams whose contribution vanishes at zero Ehrenfest time. For these contributions, the periodic orbits in Figs. 5 and 6 become important and we now view those diagrams as trajectories that have an encounter with the periodic orbit, rather than with each other. For example, in Fig. 6 we could start with the solid trajectory, which passes from channel $c$ to $d$, and the dashed trajectory from $a$ to $b$ and build the rest of the diagram from those starting points and the periodic orbit. Both of those trajectories encounter the periodic orbit once. In the semiclassical treatment of Fig. 6 above, it was implicitly assumed that these encounters occur at the same point along the periodic orbit. The resulting three-encounter can therefore be considered as an "aligned" three-encounter, but for the further Ehrenfest time dependence we also need to consider the situation where the two encounters with the periodic orbit occur at different points along the periodic orbit but still overlap. In this case we have a "non-aligned" three-encounter, while when the encounters no longer overlap we return to the two separate two-encounters of Fig. 5.

We now derive the Ehrenfest-time dependence of a nonaligned three-encounter, whose base trajectories are depicted in Fig. 7(a). In order to obtain the complete trajectory quadruplet that contributes in Eq. (7) we first need to include an additional traversal of the periodic orbit with one of the base trajectories to obtain the original trajectory pair [which has positive action in Eq. (7)]. The partner pair (with negative action) is then created by including the extra traversal of the periodic orbit with the other base trajectory. In this way we recover a diagram like Fig. 6(a) from Fig. 7(a) and a small action difference (the action of the periodic orbit itself cancels). After writing the contribution of a nonaligned three-encounter
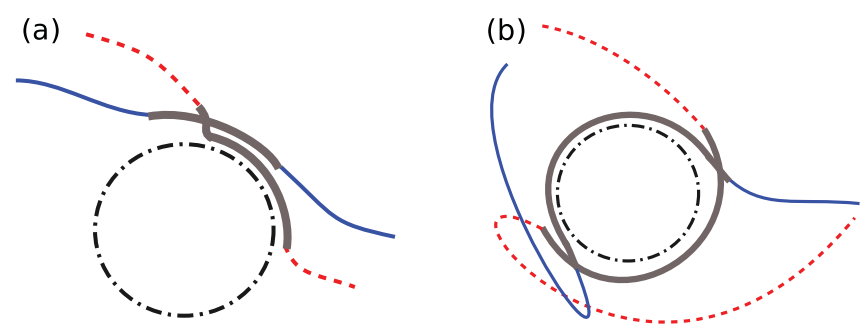

FIG. 7. (Color online) Periodic orbit encounters that contribute only for nonzero Ehrenfest time. (a) The base trajectories for a nonaligned three-encounter and (b) for encounters that overlap at both ends thus enclosing the periodic orbit (depicted dashed dotted). The encounter stretches are shown thick (gray), while the links connecting the encounter stretches to the opening are indicated by solid (blue) and dashed (red) lines. To obtain the complete quadruplet of trajectories (with a small action difference), an additional traversal of the periodic orbit must be included with one of the base trajectories and included with the other base trajectory for the partner trajectories.

in an analogous way as for the aligned three-encounter, as explained in Eqs. (13)-(15), as a product of link and encounter contributions, it can be evaluated by making use of the results for $K_{2}$ in the Appendix of Ref. 8. Of course, with the tunnel barriers, the dwell times must be modified compared to Ref. 8: The link dwell time is modified by the factor $1 / \Gamma$, while the parts of the encounter where only one trajectory is correlated to the periodic orbit - these contributions are called fringes in Ref. 8- have the factor $1 / p_{2}$ since we have two stretches close to each other. Likewise, when both trajectories are correlated with the periodic orbit we have three stretches in total and the corresponding factor $1 / p_{3}$. With these corrections, the contribution is

$$
\begin{aligned}
& \operatorname{var} G^{[7(a)]} \\
& \quad=\frac{4 N_{L}^{2} N_{R}^{2}}{\left(N_{L}+N_{R}\right)^{4}} \frac{p_{2}^{2}}{\Gamma\left(2 p_{2}-p_{3}\right)}\left(e^{-p_{3} \tau_{E} / \tau_{D}}-e^{-2 p_{2} \tau_{E} / \tau_{D}}\right) .
\end{aligned}
$$

In order to proceed to the additional diagrams that arise from touching the tunnel barriers, we first reconsider this contribution in detail along the lines of Ref. 17. As for Eqs. (13)-(15), we start with the weight function for two base trajectories encountering a periodic orbit, as explained in Refs. 6 and 8,

$$
\begin{aligned}
w(\boldsymbol{s}, \boldsymbol{u})= & \int_{0}^{T_{1}-t_{\mathrm{enc}, 1}} d t_{1} \int_{0}^{T_{2}-t_{\mathrm{enc}, 2}} d t_{2} \frac{1}{\Omega^{2}} \\
& \times \frac{1}{t_{\mathrm{enc}, 1} t_{\mathrm{enc}, 2}} \int d \tau_{p} \int d t^{\prime} .
\end{aligned}
$$

Here $t_{1}$ and $t_{2}$ are the durations of the links of the base trajectories that connect the periodic orbit encounters to the lead, while $\tau_{p}$ is the period of the periodic orbit. The integral over the period corresponds to summing over all periodic orbits that can be encountered. The encounter times are as in Eq. (14), but using the stable and unstable distances between the encounter stretches and the periodic orbit itself. Finally, $t^{\prime}$ measures the time difference between the midpoints of the two encounter stretches in Fig. 7, and the $t^{\prime}$ integral covers the different arrangements of the stretches relative to each other. 
The limits of the two last integrals in Eq. (27) are not given as they depend on the specific configuration considered below. The first two integrals in Eq. (27) can again be transformed, together with the integrals from the sum rule (9), into a product of link and encounter contributions. The general expression for the contributions to the variance from diagrams containing an enclosed periodic orbit that does not touch the lead becomes

$$
\begin{aligned}
\operatorname{var} G^{\mathrm{po}}= & \frac{\Gamma^{4} N_{L}^{2} N_{R}^{2}}{T_{H}^{2}} \prod_{i=1}^{4} \int_{0}^{\infty} d t_{i} e^{-\Gamma t_{i} / \tau_{D}} \int_{-c}^{c} d \boldsymbol{s} d \boldsymbol{u} \frac{1}{\Omega^{2}} \\
& \times \frac{e^{(i / \hbar) \boldsymbol{s} u}}{t_{\mathrm{enc}, 1} t_{\mathrm{enc}, 2}} \int d \tau_{p} \int d t^{\prime} e^{-P\left(\tau_{p}, t_{\mathrm{enc}, 1}, t_{\mathrm{enc}, 2}, t^{\prime}\right)} .
\end{aligned}
$$

Like the limits of the $\tau_{p}$ and $t^{\prime}$ integrals, the function $P$ determining the survival probability along the periodic orbit (and the encountering trajectory stretches) will be specified for each contribution separately below.

For example, for the nonaligned three-encounter, we can arrange the different alignments in terms of the durations of the two encounters. We let $t_{\text {enc,max }}$ denote the longer encounter and $t_{\text {enc,min }}$ the shorter. In Fig. 7(a), we do not yet allow the encounter stretches to surround the periodic orbit so we impose the restriction $\tau_{p}>t_{\mathrm{enc} \text {, max }}$. Then we can first consider the case where the shorter encounter lies inside the longer. We will also refer to this case later as a "generalized" three-encounter. The time difference between the midpoints of the encounters therefore satisfies $\left|t^{\prime}\right|<\left(t_{\text {enc, max }}-t_{\text {enc, min }}\right) / 2$, while the survival probability function in Eq. (28) is given by

$$
P=\frac{\Gamma\left(\tau_{p}-t_{\mathrm{enc}, \max }\right)}{\tau_{D}}+\frac{p_{2}\left(t_{\mathrm{enc}, \max }-t_{\mathrm{enc}, \min }\right)}{\tau_{D}}+\frac{p_{3} t_{\mathrm{enc}, \min }}{\tau_{D}} .
$$

The different terms simply correspond to the parts of the periodic orbit, which are followed by one, two, and three trajectory stretches, respectively. As this survival probability is independent of $t^{\prime}$, the $t^{\prime}$ integral simply yields $\left(t_{\mathrm{enc}, \max }-t_{\mathrm{enc}, \min }\right)$. Performing the remaining integrals, we obtain

$$
-\frac{2 N_{L}^{2} N_{R}^{2}}{\left(N_{L}+N_{R}\right)^{4}} \frac{p_{3}-2 p_{2}}{\Gamma} e^{-p_{3} \tau_{E} / \tau_{D}} .
$$

Of course, the range of $t^{\prime}$ includes 0 , the case in which the encounters are perfectly aligned. Equation (30) therefore includes the contribution of the three-encounter in Fig. 6(a), and for just the nonaligned three-encounter we subtract Eq. (22) to give

$$
\frac{4 N_{L}^{2} N_{R}^{2}}{\left(N_{L}+N_{R}\right)^{4}} \frac{p_{2}}{\Gamma} e^{-p_{3} \tau_{E} / \tau_{D}} .
$$

Now we consider the case in which the shorter encounter is no longer fully inside the longer, but where the two encounters still overlap. This can be further separated according to the total length of the encounters, $t_{\mathrm{encs}}=t_{\mathrm{enc}, 1}+t_{\mathrm{enc}, 2}$, compared to the periodic orbit. When $\tau_{p}>t_{\mathrm{encs}}$, we have the range

$$
\left|t^{\prime}\right| \in\left[\left(t_{\text {enc, max }}-t_{\text {enc, min }}\right) / 2, t_{\text {encs }} / 2\right]
$$

and the survival probability

$$
\begin{aligned}
P= & \frac{\Gamma \tau_{p}}{\tau_{D}}+\frac{\left(p_{2}-\Gamma\right)}{\tau_{D}}\left(\frac{t_{\text {encs }}}{2}+\left|t^{\prime}\right|\right) \\
& +\frac{\left(p_{3}-p_{2}\right)}{\tau_{D}}\left(\frac{t_{\text {encs }}}{2}-\left|t^{\prime}\right|\right),
\end{aligned}
$$

where the terms are now expressed as corrections due to additional correlated stretches. Integrating the exponential depending on this survival probability in Eq. (28) with respect to $t^{\prime}$ yields

$$
\begin{aligned}
& \frac{2 \tau_{D}}{p_{3}-2 p_{2}+\Gamma}\left(e^{-\left[\left(p_{2}-\Gamma\right) t_{\mathrm{encs}}+\Gamma \tau_{p}\right] / \tau_{D}}\right. \\
& \left.-e^{-\left[p_{3} t_{\mathrm{enc}, \min }+p_{2}\left(t_{\mathrm{enc}, \max }-t_{\mathrm{enc}, \min }\right)+\Gamma\left(\tau_{p}-t_{\mathrm{en}, \max }\right)\right] / \tau_{D}}\right) .
\end{aligned}
$$

Performing the remaining integrals in Eq. (28) leads to

$$
\begin{aligned}
& \frac{4 N_{L}^{2} N_{R}^{2}}{\left(N_{L}+N_{R}\right)^{4}} \frac{1}{\Gamma\left(p_{3}-2 p_{2}+\Gamma\right)} \\
& \quad \times\left[p_{2}^{2} e^{-2 p_{2} \tau_{E} / \tau_{D}}-p_{2}\left(p_{3}-p_{2}-\Gamma\right) e^{-\left(p_{3}+\Gamma\right) \tau_{E} / \tau_{D}}\right]
\end{aligned}
$$

If the encounters are longer than the periodic orbit, $t_{\text {enc, max }}<\tau_{p}<t_{\text {encs }}$, but we still do not allow them to overlap at both ends, we have the restriction

$$
\left|t^{\prime}\right| \in\left[\left(t_{\mathrm{enc}, \max }-t_{\mathrm{enc}, \min }\right) / 2, \tau_{p}-t_{\mathrm{encs}} / 2\right],
$$

while the survival probability remains as in Eq. (33). The $t^{\prime}$ integral yields

$$
\begin{aligned}
& \frac{2 \tau_{D}}{p_{3}-2 p_{2}+\Gamma}\left(e^{-\left[\left(p_{3}-p_{2}\right) t_{\text {encs }}+\left(2 p_{2}-p_{3}\right) \tau_{p}\right] / \tau_{D}}\right. \\
& \left.-e^{-\left[p_{3} t_{\text {enc, } \min }+p_{2}\left(t_{\text {enc, } \max }-t_{\text {enc, } \min }\right)+\Gamma\left(\tau_{p}-t_{\text {enc, } m a x}\right)\right] / \tau_{D}}\right),
\end{aligned}
$$

which finally leads to

$$
\begin{aligned}
& \frac{4 N_{L}^{2} N_{R}^{2}}{\left(N_{L}+N_{R}\right)^{4}}\left\{\frac{1}{\left(2 p_{2}-p_{3}\right)\left(p_{3}-2 p_{2}+\Gamma\right)}\right. \\
& \quad \times\left[\left(p_{3}-p_{2}\right) p_{2} e^{-p_{3} \tau_{E} / \tau_{D}}-p_{2}^{2} e^{-2 p_{2} \tau_{E} / \tau_{D}}\right] \\
& -\frac{1}{\Gamma\left(p_{3}-2 p_{2}+\Gamma\right)}\left[\left(p_{3}-p_{2}\right) p_{2} e^{-p_{3} \tau_{E} / \tau_{D}}\right. \\
& \left.\left.-p_{2}\left(p_{3}-p_{2}+\Gamma\right) e^{-\left(p_{3}+\Gamma\right) \tau_{E} / \tau_{D}}\right]\right\} .
\end{aligned}
$$

As can be easily checked, the sum of the contributions of the nonaligned three-encounter in Eqs. (31), (35), and (38) equals the contribution calculated directly in Eq. (26). The reason why we have discussed this more complicated route is that we can use it to easily calculate the contributions when we start to shrink links and allow the encounter to touch the tunnel barriers. For example, if we remove one link, then by performing analogous steps as explained before Eq. (17), we obtain

$$
\begin{aligned}
\operatorname{var} G^{[7(a)-1 l]}= & \frac{8 N_{L}^{2} N_{R}^{2}}{\left(N_{L}+N_{R}\right)^{4}} \frac{p_{2}(1-\Gamma)}{\left(2 p_{2}-p_{3}\right)}[1+(1-\Gamma)] \\
& \times\left(e^{-2 p_{2} \tau_{E} / \tau_{D}}-e^{-p_{3} \tau_{E} / \tau_{D}}\right),
\end{aligned}
$$

where in the square brackets, the 1 results from configurations where the enclosed periodic orbit touches the tunnel barrier where only one encounter stretch is correlated with the orbit, and the $(1-\Gamma)$ from configurations where the periodic 
orbit touches the tunnel barrier while both encounter stretches are correlated with it. Likewise, if two links connecting different stretches to the lead are removed, we obtain the contribution

$$
\begin{aligned}
\operatorname{var} G^{[7(a)-2 l]}= & \frac{4 N_{L}^{2} N_{R}^{2}}{\left(N_{L}+N_{R}\right)^{4}} \frac{\Gamma(1-\Gamma)^{2}(2-\Gamma)^{2}}{\left(2 p_{2}-p_{3}\right)} \\
& \times\left(e^{-p_{3} \tau_{E} / \tau_{D}}-e^{-2 p_{2} \tau_{E} / \tau_{D}}\right) .
\end{aligned}
$$

Next we turn to the configuration in Fig. 7(b) with encounter stretches overlapping at both ends. We still have $t_{\text {enc, max }}<$ $\tau_{p}<t_{\text {encs }}$, but a different restriction on $t^{\prime}$ :

$$
\left|t^{\prime}\right| \in\left[\tau_{p}-t_{\mathrm{encs}} / 2, \tau_{p} / 2,\right] .
$$

The survival probability is again independent of $t^{\prime}$, and given by

$$
P=\frac{\left(p_{3}-p_{2}\right) t_{\mathrm{encs}}}{\tau_{D}}+\frac{\left(2 p_{2}-p_{3}\right) \tau_{p}}{\tau_{D}}
$$

so that the $t^{\prime}$ integral yields $\left(t_{\mathrm{encs}}-\tau_{p}\right)$. Performing the remaining integrals we obtain

$$
\begin{aligned}
\operatorname{var} G^{[7(b)]}= & \frac{2 N_{L}^{2} N_{R}^{2}}{\left(N_{L}+N_{R}\right)^{4}}\left[\frac{p_{2}^{2}}{\left(2 p_{2}-p_{3}\right)^{2}}\right. \\
& \times\left(e^{-2 p_{2} \tau_{E} / \tau_{D}}-e^{-p_{3} \tau_{E} / \tau_{D}}\right) \\
& \left.+\frac{p_{2}\left(p_{3}-p_{2}\right) \tau_{E}}{\left(2 p_{2}-p_{3}\right) \tau_{D}} e^{-p_{3} \tau_{E} / \tau_{D}}\right] .
\end{aligned}
$$

Again the encounter stretches can be brought to the lead by first removing one link,

$$
\begin{aligned}
\operatorname{var} G^{[7(b)-1 l]}= & \frac{8 N_{L}^{2} N_{R}^{2}}{\left(N_{L}+N_{R}\right)^{4}}\left[\frac{p_{2} \Gamma(1-\Gamma)^{2}}{\left(2 p_{2}-p_{3}\right)^{2}}\right. \\
& \times\left(e^{-p_{3} \tau_{E} / \tau_{D}}-e^{-2 p_{2} \tau_{E} / \tau_{D}}\right) \\
& \left.-\frac{p_{3} \Gamma(1-\Gamma)^{2} \tau_{E}}{2\left(2 p_{2}-p_{3}\right) \tau_{D}} e^{-p_{3} \tau_{E} / \tau_{D}}\right],
\end{aligned}
$$

and second by removing two links connecting different stretches to the opening,

$$
\begin{aligned}
\operatorname{var} G^{[7(b)-2 l]}= & \frac{8 N_{L}^{2} N_{R}^{2}}{\left(N_{L}+N_{R}\right)^{4}}\left[\frac{\Gamma^{2}(1-\Gamma)^{4}}{\left(2 p_{2}-p_{3}\right)^{2}}\right. \\
& \times\left(e^{-2 p_{2} \tau_{E} / \tau_{D}}-e^{-p_{3} \tau_{E} / \tau_{D}}\right) \\
& \left.+\frac{\Gamma^{2}(1-\Gamma)^{4} \tau_{E}}{\left(2 p_{2}-p_{3}\right) \tau_{D}} e^{-p_{3} \tau_{E} / \tau_{D}}\right] .
\end{aligned}
$$

\section{Touching both leads}

As the durations of the encounters are of the order of the Ehrenfest time, for vanishing Ehrenfest time we only considered the situation where the encounter stretches could be partially reflected from the tunnel barriers in one lead. However, for increasing Ehrenfest time configurations where encounter stretches are partially reflected from the tunnel barriers in both leads, i.e., where they touch the opening at both ends, become important. If one of the two-encounters in Fig. 4 were to be partially reflected at both ends then one of the links between the two encounters would need to tunnel through the barrier and exit the system so that the rest of the diagram could not be completed. In Figs. 5 and 6, however, as long as the trajectory stretches which follow the periodic orbit are reflected at the tunnel barriers and remain in the system, we can allow the other links to tunnel through the barrier and exit the system. For the base trajectories in Fig. 7(a) this means we can allow both links of one of the base trajectories, on either side of the same encounter, to shrink into the start and end lead. We start with the configuration in Fig. 5. We first note that the contributions of the different orbit parts in Eq. (7) are multiplicative. ${ }^{32}$ We can therefore reconnect the orbits in such a way that they split into parts whose contributions have previously been calculated. First there is the remaining base trajectory, with a two-encounter with the enclosed periodic orbit and two links connecting it to the opening. This contributes the factor

$$
-\frac{N_{L} N_{R}}{\left(N_{L}+N_{R}\right)^{2}} p_{2} e^{-p_{2} \tau_{E} / \tau_{D}} .
$$

The rest of the diagram involves the periodic orbit itself and the encountering stretch that tunnels through to start and end in the leads. The contribution is calculated by summing over all enclosed periodic orbits, using the sum rule (9). Remember that when allowing the encounter to move into the lead an additional time integral occurred measuring the part of the stretch that lies still inside the system. Now there are two time integrations representing the amount of the encounter that is cut short in each of the two leads. The first time integral cancels $t_{\mathrm{enc}, i}$, while the second essentially yields a factor [1 $\left.\exp \left(-p_{2} t_{\mathrm{enc}, i} / \tau_{D}\right)\right]$. For the details of the calculation we refer to Refs. 17 and 27. This part of the diagram then contributes

$$
\frac{N_{L} N_{R}}{\left(N_{L}+N_{R}\right)^{2}} \frac{(1-\Gamma)^{2}}{p_{2}}\left(1-e^{-p_{2} \tau_{E} / \tau_{D}}\right),
$$

so that this configuration of Fig. 5 altogether yields

$$
\begin{aligned}
\operatorname{var} & G^{[5-2 l(s)]} \\
& =-\frac{4 N_{L}^{2} N_{R}^{2}}{\left(N_{L}+N_{R}\right)^{4}}(1-\Gamma)^{2} e^{-p_{2} \tau_{E} / \tau_{D}}\left(1-e^{-p_{2} \tau_{E} / \tau_{D}}\right),
\end{aligned}
$$

where the " $(s)$ " in the superscript denotes that the two links were removed along the same base trajectory from the same encounter stretch. One factor 2 in the last equation derives again from the mirror symmetry of this configuration explained after Eq. (21), the other from the fact that each of the two stretches can touch the opening at both ends.

Furthermore, one of the links of the other base trajectory may also be shrunk so that the other encounter tunnels into the lead at one end. This contribution is

$\operatorname{var} G^{[5-3 l]}=\frac{8 N_{L}^{2} N_{R}^{2}}{\left(N_{L}+N_{R}\right)^{4}} \frac{\Gamma(1-\Gamma)^{3}}{p_{2}} e^{-p_{2} \tau_{E} / \tau_{D}}\left(1-e^{-p_{2} \tau_{E} / \tau_{D}}\right)$.

Removing all four links, so that both encounter stretches on the base trajectories tunnel into the leads at both ends, is also possible. The contribution is simply given by the square of Eq. (47).

We can repeat this treatment for the aligned three-encounter of Fig. 6. If one encounter stretch tunnels into the leads at both 
ends we obtain

$$
\operatorname{var} G^{[6-2 l(s)]}=\frac{8 N_{L}^{2} N_{R}^{2}}{\left(N_{L}+N_{R}\right)^{4}} \frac{\Gamma(1-\Gamma)^{4}}{p_{3}}\left(1-e^{-p_{3} \tau_{E} / \tau_{D}}\right),
$$

where, because of the alignment and proximity of the two encounter stretches, the periodic orbit and the other encounter stretch must be backreflected at the tunnel barriers. Allowing the ends of the second encounter stretch to progressively tunnel through into the leads as well, we have, if it tunnels into the lead at one end,

$\operatorname{var} G^{[6-3 l]}=\frac{4\left(N_{L} N_{R}^{2}+N_{L}^{2} N_{R}\right)}{\left(N_{L}+N_{R}\right)^{3}} \frac{\Gamma^{2}(1-\Gamma)^{3}}{p_{3}}\left(1-e^{-p_{3} \tau_{E} / \tau_{D}}\right)$,

and at both ends

$$
\operatorname{var} G^{[6-4 l]}=\frac{2 N_{L} N_{R}}{\left(N_{L}+N_{R}\right)^{2}} \frac{\Gamma^{3}(1-\Gamma)^{2}}{p_{3}}\left(1-e^{-p_{3} \tau_{E} / \tau_{D}}\right) .
$$

Such configurations can be also considered for the diagrams in Fig. 7 and their contributions can be calculated analogously. To simplify the results, however, we will later perform an expansion of the contributions in powers of the Ehrenfest time and only retain terms up to linear order. As the results for the diagrams of Fig. 7 are all of higher order in $\tau_{E}$ than the linear one, we will not focus on their explicit form here.

\section{Encounter fringes}

A further effect, and one that actually causes the independence of the conductance variance of the Ehrenfest time for $\Gamma=1$, are correlations during fringes near periodic orbits. Encounter fringes refer to regions where the two base trajectories that encounter the periodic orbit are correlated with each other, but are no longer correlated with the periodic orbit itself; see Fig. 8 for a schematic depiction.

As the two base trajectories leave the periodic orbit correlated with each other, we can consider the encounters with the periodic orbit to be aligned. The encounter of both trajectories with the periodic orbit has length $t_{\mathrm{enc}}$ while the

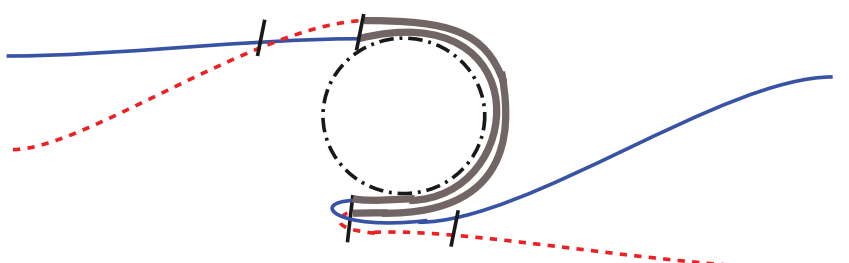

FIG. 8. (Color online) A periodic orbit encounter with fringe correlations, i.e., correlations between the two trajectories that encounter the periodic orbit (shown dashed dotted) with each other, but not with the periodic orbit itself. The fringe regions are indicated by the black lines perpendicular to the trajectories. The encounter stretches are shown thick (gray), while the links connecting the encounter stretches to the opening are indicated by solid (blue) and dashed (red) lines. fringes, during which the base trajectories are correlated, have lengths $t_{s}$ and $t_{u}$ where the subscripts refer to the fact that the stable and unstable distances between the base trajectories must be small for them to remain correlated. Here, we treat the case in which the encounter length is shorter than the periodic orbit. By this we generalize the calculation of Ref. 6 to $\Gamma \neq 1$. There it was shown that the contribution derived from Eq. (28) can be expressed as

$$
\begin{aligned}
\operatorname{var} G^{[8]}= & \frac{2 N_{L}^{2} N_{R}^{2} c^{2} \lambda}{(\pi \hbar)^{2}\left(N_{L}+N_{R}\right)^{4}} \int_{1-b}^{b-1} d s^{\prime} d u^{\prime} \int_{0}^{1} d u u \\
& \times \int_{t_{\mathrm{enc}}}^{\infty} d \tau_{p} e^{-\left[\left(p_{3}-\Gamma\right) t_{\mathrm{enc}}+\Gamma \tau_{p}\right] / \tau_{D}} \\
& \times\left(\left|\frac{s^{\prime}}{b-1}\right|^{p_{2} / \lambda \tau_{D}}-1\right)\left(\left|\frac{u^{\prime}}{b-1}\right|^{p_{2} / \lambda \tau_{D}}-1\right) \\
& \times \cos \left[\frac{c^{2} u\left(s^{\prime}-u^{\prime}\right)}{\hbar}\right],
\end{aligned}
$$

where $b$ is again a classical constant of order unity. The stable and unstable coordinates $u, s^{\prime}, u^{\prime}$ occurring in Eq. (53) are defined as in Ref. 6 and are slightly different than the $s, \boldsymbol{u}$ before in this paper, especially in Eq. (28). To understand the definition in Ref. 6 note first that correlation effects away from the periodic orbits only become important when the encountering orbits approach the periodic orbit together at one point and likewise leave together at a later point. At these two points we now place the Poincaré surfaces of sections. This situation is illustrated in Fig. 9. In the first section we first measure the distance $u$ in the unstable direction between the two approaching orbits and the central periodic one. This distance can be considered equal for the two approaching trajectories as they later leave the dashed-dotted orbit at the same point. The distance in the stable direction measured in the first section of the two approaching orbits with respect to the central periodic orbit is $c$ as where this distance falls below $c$ defines the starting point of the encounter. Second we determine in the first section the distance $s^{\prime}$ in the stable

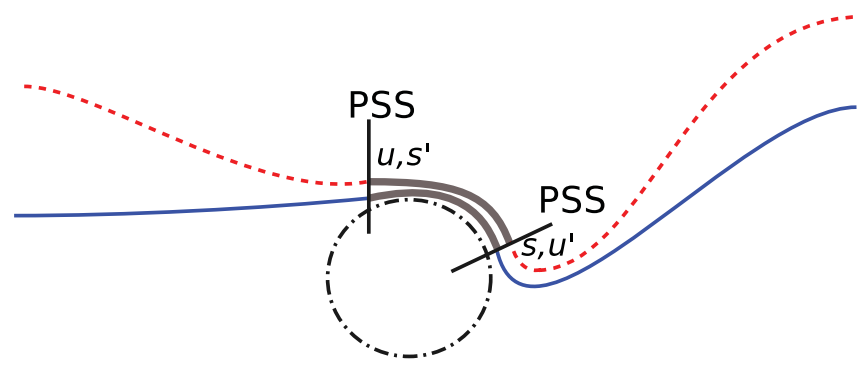

FIG. 9. (Color online) Illustration of the definition of the coordinates $u, s^{\prime}, u^{\prime}$ used in Eq. (53). The two orbits indicated by the full (blue) and dashed (red) line are assumed to approach and leave the central dashed-dotted periodic orbit together at one point. At these two points the Poincaré surfaces of sections (PSSs) are placed. In the first section first the distance $u$ in the unstable direction between the two approaching orbits and the central periodic one is measured. Second the distance $s^{\prime}$ in the stable direction between the two approaching orbits is determined. Analogously in the second section the distance $s$ of the two approaching orbits with respect to the central periodic orbit and the distance $u^{\prime}$ of these two orbits is measured. The encounter stretches are again indicated by thick (gray) lines. 
direction between the two approaching orbits. Analogously in the second section the distance $s$ of the two departing orbits with respect to the central periodic orbit is measured. The unstable distance is again $c$ so that the encounter time $t_{\mathrm{enc}}$ of the approaching orbits with the central periodic orbit is given by $t_{\text {enc }}=1 / \lambda \ln (c /|u|)=1 / \lambda \ln (c /|s|)$, then we have $|u|=|s|$ and we do not need to consider $s$ as an additional variable in Eq. (53). Finally the distance $u^{\prime}$ in the unstable direction between the two orbits departing from the central periodic orbit is determined. The two primed coordinates $s^{\prime}$ and $u^{\prime}$ characterize the time the encountering orbits remain correlated before and after they are correlated with the periodic orbit, respectively.

In Eq. (53), we included the correct dwell times for the different parts of the trajectory: $\tau_{D} / \Gamma$ for the isolated links, $\tau_{D} / p_{2}$ for the fringes, and $\tau_{D} / p_{3}$ for the encounter stretches correlated with the periodic orbit. Following the steps in Ref. 6, this contribution evaluates to

$$
\begin{aligned}
\operatorname{var} G^{[8]}= & \frac{2 N_{L}^{2} N_{R}^{2}}{\left(N_{L}+N_{R}\right)^{4}} \frac{p_{2}^{2}}{\Gamma\left(2 p_{2}-p_{3}\right)} \\
& \times\left(e^{-p_{3} \tau_{E} / \tau_{D}}-e^{-2 p_{2} \tau_{E} / \tau_{D}}\right) .
\end{aligned}
$$

Without tunnel barriers, as shown in Ref. 6, further contributions can be obtained when the fringes start in the leads. Similarly, with tunnel barriers, we can have the fringes tunnel into the leads. This leads to an additional time integral over the duration of the fringe that remains inside the system. When one fringe tunnels into the lead, two links are removed and the contribution becomes

$$
\begin{aligned}
\operatorname{var} G^{[8-2 l(s)]}= & -\frac{2\left(N_{L}^{2} N_{R}+N_{L} N_{R}^{2}\right)}{\left(N_{L}+N_{R}\right)^{3}} \frac{\Gamma p_{2}}{\left(2 p_{2}-p_{3}\right)} \\
& \times\left(e^{-p_{3} \tau_{E} / \tau_{D}}-e^{-2 p_{2} \tau_{E} / \tau_{D}}\right) .
\end{aligned}
$$

The " $(s)$ " in the superscript refers to the fact that the links were removed from the same side of the encounter. When both fringes and all four links are removed, we have

$$
\begin{aligned}
\operatorname{var} G^{[8-4 l]}= & \frac{2 N_{L} N_{R}}{\left(N_{L}+N_{R}\right)^{2}} \frac{\Gamma^{3}}{\left(2 p_{2}-p_{3}\right)} \\
& \times\left(e^{-p_{3} \tau_{E} / \tau_{D}}-e^{-2 p_{2} \tau_{E} / \tau_{D}}\right) .
\end{aligned}
$$

With the tunnel barriers though, new possibilities also arise. For example, when a fringe tunnels into the lead, one of the trajectories could be backscattered, as for the two-encounters previously. Similarly to Eq. (17), we obtain here

$$
\begin{aligned}
\operatorname{var} G^{[8-1 l]}= & -\frac{8 N_{L}^{2} N_{R}^{2}}{\left(N_{L}+N_{R}\right)^{4}} \frac{(1-\Gamma) p_{2}}{\left(2 p_{2}-p_{3}\right)} \\
& \times\left(e^{-p_{3} \tau_{E} / \tau_{D}}-e^{-2 p_{2} \tau_{E} / \tau_{D}}\right) .
\end{aligned}
$$

Furthermore, we can move additionally one fringe into the lead yielding

$$
\begin{aligned}
\operatorname{var} G^{[8-3 l]}= & \frac{4\left(N_{L} N_{R}^{2}+N_{L}^{2} N_{R}\right)}{\left(N_{L}+N_{R}\right)^{3}} \frac{(1-\Gamma) \Gamma^{2}}{\left(2 p_{2}-p_{3}\right)} \\
& \times\left(e^{-p_{3} \tau_{E} / \tau_{D}}-e^{-2 p_{2} \tau_{E} / \tau_{D}}\right) .
\end{aligned}
$$

If both fringes are partially backscattered at the leads, we have the contribution

$$
\begin{aligned}
\operatorname{var} G^{[8-2 l]}= & \frac{8 N_{L}^{2} N_{R}^{2}}{\left(N_{L}+N_{R}\right)^{4}} \frac{\Gamma(1-\Gamma)^{2}}{\left(2 p_{2}-p_{3}\right)} \\
& \times\left(e^{-p_{3} \tau_{E} / \tau_{D}}-e^{-2 p_{2} \tau_{E} / \tau_{D}}\right) .
\end{aligned}
$$

Next we can allow the periodic orbit itself, rather than the fringes, to touch the lead and be reflected from the tunnel barriers. The encounter stretches may then both tunnel into the lead, or one may be reflected. We recall that the case in which both are reflected is already included in the survival probability of the encounter. One fringe is therefore removed from the systems, while the fringe that remains could then be inside the system, tunnel into the lead, or be partially backreflected at the opening. If the periodic orbit touches both leads during the encounter, we return to the situation in the previous subsection, so here we only consider the cases in which at least some of the second fringe remains.

The removal of one fringe entirely implies the following changes in Eq. (53): With only one fringe time left, we only have one of the factors in the third line of Eq. (53). Also the encounter time is replaced by an integration variable (with a range from 0 to $t_{\mathrm{enc}}$ ) that accounts for the amount of the encounter left after the periodic orbit is reflected from the tunnel barriers. Performing, with these changes, again the steps in Ref. 6 analogous to those following Eq. (53) above, we obtain the contributions for the various different cases. First, when the remaining fringe lies inside the system, we could have the encounter (which touches the lead) partially reflected,

$$
\begin{aligned}
\operatorname{var} G^{[8-1 f-1 l]}= & -\frac{4 N_{L}^{2} N_{R}^{2}}{\left(N_{L}+N_{R}\right)^{4}} \frac{(1-\Gamma)^{2} p_{2}}{p_{3}} \\
& \times\left(1-e^{-p_{3} \tau_{E} / \tau_{D}}\right) e^{-p_{2} \tau_{E} / \tau_{D}},
\end{aligned}
$$

where " $-1 f-1 l$ " refers to the fact that one fringe and one link were removed. Likewise, both encounter stretches could tunnel directly into the lead so that two links are removed on the same side of the encounter,

$$
\begin{aligned}
\operatorname{var} G^{[8-1 f-2 l(s)]}= & -\frac{\left(N_{L} N_{R}^{2}+N_{L}^{2} N_{R}\right)}{\left(N_{L}+N_{R}\right)^{3}} \frac{(1-\Gamma) p_{2} \Gamma}{p_{3}} \\
& \times\left(1-e^{-p_{3} \tau_{E} / \tau_{D}}\right) e^{-p_{2} \tau_{E / \tau}}
\end{aligned}
$$

The remaining fringe could also be partially reflected from the tunnel barriers. For the encounter stretches we have the same two possibilities as above. This leads to

$$
\begin{aligned}
\operatorname{var} G^{[8-1 f-2 l]}= & \frac{8 N_{L}^{2} N_{R}^{2}}{\left(N_{L}+N_{R}\right)^{4}} \frac{(1-\Gamma)^{3} \Gamma}{p_{3}} \\
& \times\left(1-e^{-p_{3} \tau_{E} / \tau_{D}}\right) e^{-p_{2} \tau_{E} / \tau_{D}},
\end{aligned}
$$

and

$$
\begin{aligned}
\operatorname{var} G^{[8-1 f-3 l(s)]}= & \frac{2\left(N_{L}^{2} N_{R}+N_{L} N_{R}^{2}\right)}{\left(N_{L}+N_{R}\right)^{3}} \frac{(1-\Gamma)^{2} \Gamma^{2}}{p_{3}} \\
& \times\left(1-e^{-p_{3} \tau_{E} / \tau_{D}}\right) e^{-p_{2} \tau_{E} / \tau_{D}} .
\end{aligned}
$$


Finally the remaining fringe can tunnel directly into the lead, giving the contributions

$$
\begin{aligned}
\operatorname{var} G^{[8-1 f-3 l]}= & \frac{2\left(N_{L}^{2} N_{R}+N_{L} N_{R}^{2}\right)}{\left(N_{L}+N_{R}\right)^{3}} \frac{(1-\Gamma)^{2} \Gamma^{2}}{p_{3}} \\
& \times\left(1-e^{-p_{3} \tau_{E} / \tau_{D}}\right) e^{-p_{2} \tau_{E} / \tau_{D}},
\end{aligned}
$$

and

$$
\begin{aligned}
\operatorname{var} G^{[8-1 f-4 l]}= & \frac{2 N_{L} N_{R}}{\left(N_{L}+N_{R}\right)^{2}} \frac{(1-\Gamma)^{2} \Gamma^{3}}{p_{3}} \\
& \times\left(1-e^{-p_{3} \tau_{E} / \tau_{D}}\right) e^{-p_{2} \tau_{E} / \tau_{D}} .
\end{aligned}
$$

\section{E. Multiple periodic orbit traversals}

Having treated all encounter configurations with periodic orbits where the encounter stretches are shorter than the enclosed periodic orbit, we now turn to the corresponding contributions where the encounter stretches are longer than the enclosed periodic orbit. In this context only diagrams with stretches that both possess the same number of traversals around the enclosed periodic orbit yield a contribution; for a justification see Ref. 6 . In this context we consider that each encounter stretch has $k$ full windings around the enclosed periodic orbit. We take here the two encounter times $t_{\mathrm{enc}, 1}, t_{\mathrm{enc}, 2}$ and the corresponding primitive times $t_{\mathrm{enc}, i}^{p} \equiv t_{\mathrm{enc}, i}-k \tau_{p}$. These primitive times are again shorter than the enclosed periodic orbit and we can now also consider the different cases depicted in Figs. 5-8, and how they can be arranged. We start with the case in which the two primitive encounter times $t_{\mathrm{enc}, i}^{p}$ do not overlap. When $k=0$, the corresponding diagram is depicted in Fig. 5. To explain the calculation we begin with Eq. (28) for $k=0$ and perform the integrals over the links $t_{i}$, and over $t^{\prime}$ where the latter integral leads to the factor $\left(\tau_{p}-t_{\text {encs }}\right)$ :

$$
\begin{aligned}
\operatorname{var} G_{k=0}^{[5]}= & \frac{N_{L}^{2} N_{R}^{2}}{\left(N_{L}+N_{R}\right)^{4}} \int_{-c}^{c} d \boldsymbol{s} d \boldsymbol{u} \frac{T_{H}^{2}}{\Omega^{2}} \frac{e^{(i / \hbar) s \boldsymbol{u}}}{t_{\mathrm{enc}, 1} t_{\mathrm{enc}, 2}} \\
& \times \int_{t_{\mathrm{encs}}}^{\infty} d \tau_{p}\left(\tau_{p}-t_{\mathrm{encs}}\right) e^{-\left[\left(p_{2}-\Gamma\right) t_{\mathrm{encs}}+\Gamma \tau_{p}\right] / \tau_{D}} .
\end{aligned}
$$

To turn to $k \neq 0, t_{\mathrm{enc}, i}$ is then replaced only inside the integrand of the $\tau_{p}$ integral by $t_{\mathrm{enc}, i}^{p}$; these times are shorter than $\tau_{p}$ by definition. The $t_{\mathrm{enc}, i}$ before the $\tau_{p}$ integral compensate the overcounting of equivalent positions of the Poincaré surface of sections by the $s_{i}, u_{i}$ integrals and thus do not need to be altered. The possibilities for placing these stretches around the periodic orbit are the same as they were for $t_{\mathrm{enc}, i}$ for $k=0$. This allows us to treat $k>0$ in essentially the same way we treated $k=0$ before. As $t_{\mathrm{enc}, i}^{p}=t_{\mathrm{enc}, i}-k \tau_{p}$, we reexpress the primitive encounter times in terms of $t_{\mathrm{enc}, i}$ and $\tau_{p}$. The limits of the $\tau_{p}$ integration are also altered for $k \neq 0$ : As the primitive encounters do not (yet) overlap, we have the condition $t_{\mathrm{enc}, 1}^{p}+$ $t_{\mathrm{enc}, 2}^{p} \geqslant \tau_{p}$ so that the lower limit is now $t_{\mathrm{encs}} /(2 k+1)$. Since we remove all $k$ complete windings of the periodic orbit from both encounter times, the shorter encounter must be $t_{\text {enc, min }} \geqslant$ $k \tau_{p}$ so that the upper limit is $t_{\mathrm{enc}, \min } / k$. Finally $p_{j}$ is replaced by $p_{2 k+j}$ (where in this context $\Gamma$ is defined as $p_{1}$ ) since during the primitive encounter stretches we have a total of $2 k+2$ correlated stretches while elsewhere we have $2 k+1$.
All these replacements finally yield

$$
\begin{aligned}
\operatorname{var} G_{\mathrm{mt}}^{[5]}= & \frac{N_{L}^{2} N_{R}^{2}}{\left(N_{L}+N_{R}\right)^{4}} \int_{-c}^{c} d \boldsymbol{s} d \boldsymbol{u} \frac{T_{H}^{2}}{\Omega^{2}} \frac{e^{(i / \hbar)} \boldsymbol{s} \boldsymbol{e}}{t_{\mathrm{enc}, 1} t_{\mathrm{enc}, 2}} \\
& \times \int_{t_{\mathrm{encs}} /(2 k+1)}^{t_{\mathrm{enc}, \min / k}} d \tau_{p}\left[(2 k+1) \tau_{p}-t_{\mathrm{encs}}\right] \\
& \times e^{-\left[\left(p_{2 k+2}-p_{2 k+1}\right)\left(t_{\mathrm{encs}}-2 k \tau_{p}\right)+p_{2 k+1} \tau_{p}\right] / \tau_{D}},
\end{aligned}
$$

where the additional index "mt" indicates that this contribution results from multiple traversals $k>0$. Performing now the remaining integrals in the same way as, for example, in Eqs. (34)-(38) we obtain this contribution. As the full expression is rather involved we just give here terms up to linear order in $\tau_{E}$ :

$\operatorname{var} G_{\mathrm{mt}}^{[5]}=-\frac{2 N_{L}^{2} N_{R}^{2}}{\left(N_{L}+N_{R}\right)^{4}} \sum_{k=1}^{\infty} \frac{\left(p_{2 k+2}-p_{2 k+1}\right) \tau_{E}}{k^{2}(2 k+1) \tau_{D}}+\mathcal{O}\left(\tau_{E}^{2}\right)$.

We also omitted here terms that are also nonzero for $p_{k}=1$ as they all cancel with the contributions below. Also in this case one link can be removed leading to a configuration where one encounter stretch touches the opening. This implies that in Eq. (67) one factor $\tau_{D} / \Gamma$ is removed, the duration of the encounter stretch that touches the tunnel barrier is replaced by an integration variable integrated from 0 to $t_{\mathrm{enc}, i}$, and additionally a factor $(1-\Gamma)^{2 k+1}$ arises to account for the probability that all the $2 k+1$ orbital parts surrounding the periodic orbit are backreflected when they hit the lead. This contribution then yields

$\operatorname{var} G_{\mathrm{mt}}^{[5-1 l]}=\frac{4 N_{L}^{2} N_{R}^{2}}{\left(N_{L}+N_{R}\right)^{4}} \sum_{k=1}^{\infty} \frac{\left(p_{2 k+2}-p_{2 k+1}\right) \tau_{E}}{k^{2}(2 k+1) \tau_{D}}+\mathcal{O}\left(\tau_{E}^{2}\right)$.

Shrinking two links connecting two different encounter stretches to the opening, we obtain (following the same steps as just described also for the other $t_{\mathrm{enc}, i}$ ) no contribution that is linear in $\tau_{E}$. Shrinking two links connecting the same encounter we need again to introduce in Eq. (67) two additional time integrals as described before Eq. (47). This also finally yields zero contribution linear in $\tau_{E}$, because the terms resulting from the two limits of the $\tau_{p}$ integral cancel. The same also holds for the following contributions.

Similar contributions are also obtained in the other cases where we give more details of the calculation in Appendix A. For a generalized three-encounter introduced around Eq. (29) we obtain the integrals given in Eq. (A1), which provide the contribution

$$
\begin{aligned}
\operatorname{var} G_{\mathrm{mt}}^{[6]}= & \frac{2 N_{L}^{2} N_{R}^{2}}{\left(N_{L}+N_{R}\right)^{4}} \sum_{k=1}^{\infty} \frac{\tau_{E}}{k(k+1) \tau_{D}} \\
& \times\left(2 p_{2 k+2}-p_{2 k+1}-p_{2 k+3}\right)+\mathcal{O}\left(\tau_{E}^{2}\right)
\end{aligned}
$$

when the encounters lie inside the system and

$$
\begin{aligned}
\operatorname{var} G_{\mathrm{mt}}^{[6-1 l]}= & -\frac{4 N_{L}^{2} N_{R}^{2}}{\left(N_{L}+N_{R}\right)^{4}} \sum_{k=1}^{\infty} \frac{\tau_{E}}{k(k+1) \tau_{D}} \\
& \times\left(2 p_{2 k+2}-p_{2 k+1}-p_{2 k+3}\right)+\mathcal{O}\left(\tau_{E}^{2}\right),
\end{aligned}
$$


when one link is removed. Additionally we can shrink both links connecting the three-encounter to the opening on one side. This amounts to removing two links, and introducing an additional integral over the part of the encounter that remains inside the system, in Eq. (A1), yielding

$$
\begin{aligned}
\operatorname{var} G_{\mathrm{mt}}^{[6-2 l]}= & \frac{2\left(N_{L} N_{R}^{2}+N_{L}^{2} N_{R}\right)}{\left(N_{L}+N_{R}\right)^{3}} \sum_{k=1}^{\infty} \frac{\tau_{E}}{k(k+1) \tau_{D}} \\
& \times\left(2 p_{2 k+2}-p_{2 k+1}-p_{2 k+3}\right)+\mathcal{O}\left(\tau_{E}^{2}\right) .
\end{aligned}
$$

Again zero contribution is obtained when more links are removed.

For a nonaligned three-encounter, when the encounter lies inside the system we obtain from Eqs. (A2) and (A3)

$$
\begin{aligned}
\operatorname{var} G_{\mathrm{mt}}^{[7(a)]}= & \frac{4 N_{L}^{2} N_{R}^{2}}{\left(N_{L}+N_{R}\right)^{4}} \sum_{k=1}^{\infty}\left[\frac{\left(p_{2 k+3}-p_{2 k+2}\right) \tau_{E}}{(k+1)(2 k+1) \tau_{D}}\right. \\
& \left.-\frac{\left(p_{2 k+2}-p_{2 k+1}\right) \tau_{E}}{k(2 k+1) \tau_{D}}\right]+\mathcal{O}\left(\tau_{E}^{2}\right),
\end{aligned}
$$

and when one link is removed,

$$
\begin{aligned}
\operatorname{var} G_{\mathrm{mt}}^{[7(a)-1 l]}= & -\frac{8 N_{L}^{2} N_{R}^{2}}{\left(N_{L}+N_{R}\right)^{4}} \sum_{k=1}^{\infty}\left[\frac{\left(p_{2 k+3}-p_{2 k+2}\right) \tau_{E}}{(k+1)(2 k+1) \tau_{D}}\right. \\
& +\frac{\left(2 p_{2 k+2}-p_{2 k+1}-p_{2 k+3}\right) \tau_{E}}{2 k(k+1) \tau_{D}} \\
& \left.-\frac{\left(p_{2 k+2}-p_{2 k+1}\right) \tau_{E}}{k(2 k+1) \tau_{D}}\right]+\mathcal{O}\left(\tau_{E}^{2}\right)
\end{aligned}
$$

Finally we treat the configuration in which the encounter stretches overlap at both ends. When $k=0$ this configuration is depicted in Fig. 7(b). With multiple traversals of the periodic orbit, the contribution given in Eq. (A4) provides, when the stretches lie inside the system,

$$
\begin{aligned}
\operatorname{var} G_{\mathrm{mt}}^{[7(b)]}= & -\frac{2 N_{L}^{2} N_{R}^{2}}{\left(N_{L}+N_{R}\right)^{4}} \sum_{k=1}^{\infty} \frac{\left(p_{2 k+3}-p_{2 k+2}\right) \tau_{E}}{(k+1)^{2}(2 k+1) \tau_{D}} \\
& +\mathcal{O}\left(\tau_{E}^{2}\right)
\end{aligned}
$$

and when one link is removed

$$
\begin{aligned}
\operatorname{var} G_{\mathrm{mt}}^{[7(b)-1 l]}= & \frac{4 N_{L}^{2} N_{R}^{2}}{\left(N_{L}+N_{R}\right)^{4}} \sum_{k=1}^{\infty} \frac{\left(p_{2 k+3}-p_{2 k+2}\right) \tau_{E}}{(k+1)^{2}(2 k+1) \tau_{D}} \\
& +\mathcal{O}\left(\tau_{E}^{2}\right) .
\end{aligned}
$$

No further contributions are obtained when taking into account fringes as in Fig. 8. This can be checked from Eq. (53) by adjusting the limits of the $\tau_{p}$ integral appropriately. Then, the terms from the two limits of the $\tau_{p}$ integral cancel to linear order in $\tau_{E}$.

\section{F. Linear Ehrenfest-time dependence}

Having calculated all the contributions we can now sum them to obtain the overall contributions to the conductance variance, to leading order in the inverse number of open channels in the leads. First we can check that all the terms after Sec. III A are zero for $\tau_{E}=0$, so that the RMT result (25) is preserved. The case that is especially interesting for the comparison with the numerics is the contribution to the conductance variance when $N_{L}=N_{R}$, for arbitrary $\Gamma$ and of linear order in $\tau_{E}$. We therefore expand the terms in Eqs. (16)-(24), (26), (39), (40), (43)-(45), (48)-(52), and (54)-(65) up to linear order in $\tau_{E} / \tau_{D}$. The first term then yields the RMT contribution, the second we obtain by adding additionally Eqs. (68)-(76). We write it as $\tau_{E} / \tau_{D}$ times a $\Gamma$-dependent prefactor $\alpha(\Gamma)$ and obtain

$$
\begin{aligned}
\operatorname{var} G_{N_{L}=N_{R}} \approx & \operatorname{var} G^{\mathrm{RMT}}+\alpha \frac{\tau_{E}}{\tau_{D}}+\mathcal{O}\left(\frac{\tau_{E}^{2}}{\tau_{D}^{2}}\right)+\mathcal{O}\left(\frac{1}{N}\right) \\
\approx & \frac{1}{8}\left[1+(1-\Gamma)^{2}\right]+\alpha \frac{\tau_{E}}{\tau_{D}}+\mathcal{O}\left(\frac{\tau_{E}^{2}}{\tau_{D}^{2}}\right) \\
& +\mathcal{O}\left(\frac{1}{N}\right)
\end{aligned}
$$

and

$$
\begin{aligned}
\alpha(\Gamma)= & \frac{\Gamma}{4}\left[(\Gamma-1)\left(7 \Gamma^{3}-6 \Gamma^{2}+4 \Gamma-2\right)+\ln \left(2 \Gamma-\Gamma^{2}\right)\right. \\
& \left.\times \frac{\Gamma^{2}(2-\Gamma)}{(1-\Gamma)}-\Gamma \operatorname{Li}_{2}\left[(1-\Gamma)^{2}\right]\right]
\end{aligned}
$$

The parameter regime for this prediction to be valid is thus $\tau_{E} / \tau_{D} \ll 1$ and $N \gg 1$. Here $\operatorname{Li}_{2}(x)$ denotes the polylogarithmic function, which arises when performing the $k$ summations above. The function $\alpha(\Gamma)$ is shown as the solid (red) line in Fig. 2.

Having derived the complete set of contributions, we now discuss the main terms that lead to the two extrema in Fig. 2. First we note that the contributions from multiply traversed periodic orbits are quite small. When $\Gamma=1$ they provide no contribution linear in $\tau_{E}$, as can be checked from Eqs. (68)(76). For most of the contributions for $\Gamma \neq 1$ the index $k$ of the $p_{k}$ 's in the sum is much larger than 1 . In this case the differences of the $p_{k}$ 's in Eqs. (68)-(76) tend to 1 and thus the contributions from these equations tend to 0 . Then the result is almost equal to the vanishing value for $\Gamma=1$.

In Fig. 2, the maximum lies in a region of small $\Gamma$. In this regime, most of the diagrams approximately cancel each other as can be seen by expanding their contributions in a Taylor series for small $\Gamma$ : The ones from diagrams containing encounters inside the system [e.g., the contribution (16)] are canceled by the contributions obtained when one and two links are shrunk [e.g., the contributions (17)-(21)]. However, this cancellation does not hold for the three-encounter with the periodic orbit (see Fig. 6). In this case we have two possibilities: First that the encounter lies inside the system or touches the lead at one end [see Eqs. (22)-(24)]. In total this leads to an Ehrenfest-time-dependent factor of $e^{-p_{3} \tau_{E} / \tau_{D}}$. Additionally, we also need to take into account the contributions from encounters touching the leads on both sides leading to an increasing contribution proportional to $1-e^{-p_{3} \tau_{E} / \tau_{D}}$ with a larger prefactor than the first one [see Eqs. (50)-(52)]. The increasing contribution from similar diagrams also partially canceled the decrease of the Fano factor and $S$-matrix correlation functions in Refs. 14 and 17 in systems without tunnel barriers. This term is usually referred to as "classical" as it also survives for $\tau_{E} \rightarrow \infty$ and increases with increasing $\tau_{E}$. Together, the two possibilities for the 
three-encounter with the periodic orbit compete to cause the first peak in Fig. 2.

In the case of the dip the factor $(1-\Gamma)$, i.e., the probability for the particle to be backreflected at the opening, is quite small. This implies that we get the main contributions from diagrams with encounters inside the system (i.e., the ones also obtained for $\Gamma=1$ ) depicted in Figs. 4-8. These together yield a negative contribution.

\section{NUMERICAL SIMULATION}

We numerically confirm our main analytical prediction, Eqs. (77) and (78). The model we use is the open kicked rotator with time-dependent Hamiltonian ${ }^{35}$

$$
\hat{H}=\frac{\left(p+p_{0}\right)^{2}}{2}+K \cos \left(x+x_{0}\right) \sum_{n=-\infty}^{\infty} \delta\left(t-n \tau_{f}\right),
$$

with $\tau_{f}$ the free flight time, which we set to 1 . Depending on the kicking strength $K$ the dynamics changes from integrable for $K=0$ to fully chaotic for $K \gtrsim 7$. In the latter regime, local exponential instability is characterized by the Lyapunov exponent

$$
\lambda=\ln \left(\frac{K}{2}\right) .
$$

The quantities $p_{0}$ and $x_{0}$ are introduced to break the Hamiltonian's two symmetries, ${ }^{35}$ and investigate different symmetry classes with $\left(p_{0}\right.$ or $\left.x_{0}=0\right)$ and without $\left(p_{0} \neq 0 \neq\right.$ $x_{0}$ ) time-reversal symmetry. The Ehrenfest time in this system is determined, up to a constant of little relevance, by $\tau_{E} \equiv$ $\lambda^{-1} \ln (M / c)$ with $M$ the size of the Hilbert space, determined by the quantization of the Hamiltonian via discretization of the coordinates as, e.g., $p_{l}=2 \pi l / M, l=1, \ldots M$. Here $c$ is again a system-dependent constant of order 1 that is of classical origin, and as such does not depend on $M$. A quantum representation of the Hamiltonian (79) is then provided by the unitary $M \times M$ Floquet matrix $U$, giving the time evolution for one iteration of the map defined by $\hat{H}$ in a time interval $\left[t_{0}, t_{0}+1\right)$. For our specific choice of the kicked rotator, the Floquet operator has matrix elements

$$
\begin{aligned}
U_{l, l^{\prime}}= & M e^{-(\pi i / M)\left[\left(l+l_{0}\right)^{2}+\left(l^{\prime}+l_{0}\right)^{2}\right]} \\
& \times \sum_{m} e^{2 \pi i m\left(l-l^{\prime}\right) / M} e^{-(i M K / 4 \pi) \cos \left[2 \pi\left(m+m_{0}\right) / M\right]}
\end{aligned}
$$

with $l_{0}=p_{0} M / 2 \pi$ and $m_{0}=x_{0} M / 2 \pi$. Transport can finally be investigated once absorbing phase-space strips are introduced to model contacts to leads. This is achieved by means of projection operators $P$. In our case of tunnel-coupled leads, the latter are $N \times M$ diagonal matrices with entries $P_{i j}=\delta_{i j} \sqrt{\Gamma}$, assuming that the system is coupled to all $N=$ $N_{L}+N_{R}$ channels with the same transparency $0<\Gamma \leqslant 1$. The scattering matrix is finally defined as ${ }^{36}$

$S(\varepsilon)=\left(1-P^{T} P\right)^{1 / 2}-P\left[\exp (-i \varepsilon)-U\left(1-P^{T} P\right)\right]^{-1} U P^{T}$.

The kicked rotator model is particularly well suited to investigate the semiclassical limit with $\tau_{E} \gtrsim \tau_{D}$, as it allows for a rather large variation of the system size and hence the

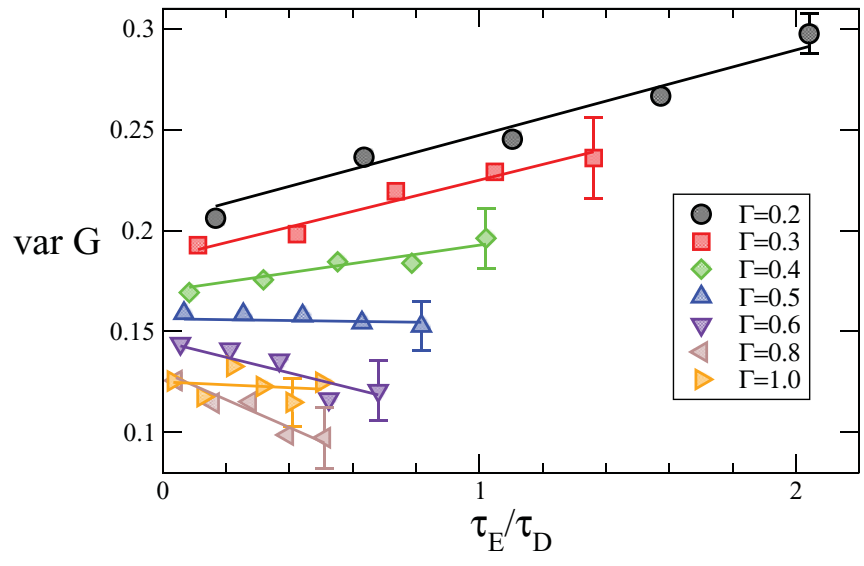

FIG. 10. (Color online) Conductance variance var $G(E)$ as a function of $\tau_{E} / \tau_{D}$ for various values of $\Gamma$. In the horizontal axis, we use $\tau_{E}=\lambda^{-1} \ln (M / c)$, with $c$ of the order 1 and $\lambda$ as defined in Eq. (80). The uncertainty in the Lyapunov exponent due to finite-time effects and the arbitrariness of the constant $c$ are fixed by requiring that analytical and numerical data agree for $\Gamma=0.2$, as described in the text. The straight lines are linear fits constrained to go to $\left[1+(1-\Gamma)^{2}\right] / 8$ at $\tau_{E}=0$. Their slopes give the parameter $\alpha$ defined in Eqs. (77) and (78). The extracted values of $\alpha$ are plotted as a function of $\Gamma$ in Fig. 2.

Ehrenfest time. Previous such investigations of the open kicked rotator are presented, e.g., in Refs. 7, 9, 37, and 38, and we refer the reader to these works for further details of the model. The variance of the conductance is calculated by varying the quasienergies $\varepsilon$ at given lead position. Averages of $\operatorname{var} G(E)$ are further performed with different lead positions.

We first show in Fig. 10 the behavior of $\operatorname{var} G(E)$ as a function of $\tau_{E} / \tau_{D}$. To reach the largest possible values of $\tau_{E} / \tau_{D}$, while still remaining in the chaotic regime $\lambda \tau_{D} \gg 1$, we take values $K=10$ and $\tau_{D}=M / 2 N=5 \Gamma$, but stress that further numerical data, which we do not show for $K=20$ and/or $\tau_{D}=10 \Gamma$, corroborate our conclusions. The Ehrenfest time is varied at fixed classical dynamics by varying $M$ as powers of 2 between 128 and 2048, with the additional value of $M=4096$ for $\Gamma=1$. We calculate $\operatorname{var} G(E)$ for 50 to 100 different values of $E$ at fixed position of the leads, and repeat the operation for 500 (for $M=128$ ) to 50 (for $M=4096$ ) different lead positions. The error bars in Fig. 10 reflect the maximal obtained spread of $\operatorname{var} G(E)$ as lead positions are changed, for each value of $\Gamma$. We clearly see $\Gamma$-dependent behaviors, as var $G(E)$ increases for small $\Gamma$ and decreases for larger $\Gamma$ until it becomes independent of $\tau_{E}$ for $\Gamma=1$. We extract the linear slope of the $\operatorname{var} G(E)$ vs $\tau_{E} / \tau_{D}$ curve via a linear fit, setting $\tau_{E}=0$ where the curves intersect the RMT universal value $\operatorname{var}(G)^{\mathrm{RMT}}=\left[1+(1-\Gamma)^{2}\right] / 8 .^{28}$ There is still an uncertainty in $\alpha$ due to an uncertainty in the precise value of the Lyapunov exponent-the latter has been found numerically to deviate in open systems from its exact value (80), which has been attributed to finite-time effects-and because of the arbitrary constant $c$ of order 1 in the definition of $\tau_{E}$. We remove this uncertainty by forcing numerical and analytical data to agree for $\Gamma=0.2$. Once this is done, there is no free parameter left. We compare the so-obtained numerical values for $\alpha$ with the analytical prediction of 
Eq. (78) in Fig. 2. The excellent agreement between numerical data and the analytical curve fully confirms our theory. We also checked, but do not show, that the linear $\tau_{E}$ dependence of $\operatorname{var} G(E)$ is halved when time-reversal symmetry is broken.

\section{CONCLUSIONS}

In this paper we determined the dependence of the conductance variance of a chaotic cavity with tunnel barriers on the tunneling probability $\Gamma$. In particular we find an Ehrenfesttime dependence for the general case $\Gamma<1$ of nonperfect coupling. We focused on the contribution that is linear in the Ehrenfest time and leading order in the inverse total number of open channels $1 / N$. We predict a nonmonotonous sinusoidal behavior with one maximum at $\Gamma \approx 0.2$ and a (roughly $50 \%$ ) deeper minimum at $\Gamma \approx 0.8$. This analytical result was derived semiclassically by systematically considering a rather large number of possible configurations, but the general behavior derives from a much smaller set. We discussed the main contributions, which lead to each of the two peaks to provide a better intuitive understanding of the overall structure. Finally, we compared these analytical predictions with numerical simulations performed for the kicked rotor (in the chaotic regime). There was good agreement between the two, with the analytical curve within the error bars of all the numerically determined data points.

Although, even at leading order, we treated a large number of possible semiclassical diagrams, they could be constructed, as we showed in this paper, in a controlled way from the diagrams that exist when the tunnel barriers are absent. This calculation could therefore possibly be extended to higherorder contributions in $1 / N$ and $\tau_{E}$, but a more natural next step would be to extend the Ehrenfest-time dependence of the (leading order in $1 / N$ ) results for the full counting statistics of transport moments ${ }^{17}$ to include tunnel barriers in the leads and to account for the extra diagrammatic possibilities that then arise.

On the numerical side, we emphasize that the agreement observed in this paper between numerics and analytics could neither be obtained concerning the analytical results for the conductance nor for shot noise in Ref. 33. Further numerical investigation in this area would therefore be highly desirable. Finally it would be interesting to check the observed phenomena experimentally. Although Ehrenfest-time effects in antidot superlattices were observed more than ten years ago, ${ }^{4}$ none of the other Ehrenfest-time dependencies predicted for chaotic systems have been checked so far experimentally. A candidate was worked out by Tian, Larkin, and Kamenev in Ref. 39 for the Ehrenfest-time dependence of weak localization in kicked rotors as being suitable for experimental verifications in the cold atomic gas context. The difficulty to observe these effects is mainly due to the small range through which the system size can be varied in quantum dots. However, with the additional parameter of the tunneling probability and in view of the expected double peak structure, the prediction in this paper could be amenable to experimental verification. In the case of electron based transport it is an interesting question of how our calculations are affected by interaction effects that are known to be especially relevant in the regime $\Gamma \ll 1{ }^{26}$ However, such effects could be avoided by considering other realizations such as microwave or cold-atom billiards.

\section{ACKNOWLEDGMENTS}

This work was funded by the Deutsche Forschungsgemeinschaft (Research Unit FOR 760) (D.W. and K.R.) and by the Alexander von Humboldt Foundation (J.K.). P.J. has been partially supported by the NSF under Grant No. DMR0706319 and by the Swiss Center of Excellence MaNEP. It is a great pleasure to thank C. Petitjean for discussions and for drawing our attention to this problem. Furthermore, we thank $\mathrm{R}$. Whitney for discussions at the early stage of this project.

\section{APPENDIX A: PERIODIC ORBIT ENCOUNTERS}

Here we give the necessary integrals for obtaining the contributions from diagrams containing enclosed periodic orbits that are surrounded $k$ times by each encounter stretch. In these expressions the link and $t^{\prime}$ integrals in Eq. (28) have already been performed, but not yet the $\tau_{p}$ and the $\boldsymbol{s}, \boldsymbol{u}$ integrals. These expressions are especially useful because they not only allow one to obtain the contributions from encounters inside the system but (via integrations with respect to the corresponding encounter times) also the contributions when encounters touch the openings.

The corresponding expression obtained for two independent two-encounters was already given in the main text in Eq. (67). In the case of a generalized three-encounter we obtain

$$
\begin{aligned}
\operatorname{var} G_{\mathrm{mt}}^{[6]}= & \frac{2 N_{L}^{2} N_{R}^{2}}{\left(N_{L}+N_{R}\right)^{4}} \int_{-c}^{c} d \boldsymbol{s} d \boldsymbol{u} \int_{t_{\mathrm{enc}, \max } /(k+1)}^{t_{\mathrm{enc}, \min } / k} d \tau_{p} \frac{T_{H}^{2}}{\Omega^{2}} \\
& \times \frac{e^{(i / \hbar) s \boldsymbol{u}}}{t_{\mathrm{enc}, 1} t_{\mathrm{enc}, 2}}\left(t_{\mathrm{enc}, \max }-t_{\mathrm{enc}, \min }\right) \\
& \times e^{-\left(p_{2 k+3}-p_{2 k+2}\right)\left(t_{\mathrm{enc}, \min }-k \tau_{p}\right) / \tau_{D}} \\
& \times e^{-\left[\left(p_{2 k+2}-p_{2 k+1}\right)\left(t_{\mathrm{enc}, \max }-k \tau_{p}\right)+p_{2 k+1} \tau_{p}\right] / \tau_{D}} .
\end{aligned}
$$

For a nonaligned three-encounter, similar to Eqs. (34) and (37), it is given first for $\tau_{p}>t_{\mathrm{enc}, 1}^{p}+t_{\mathrm{enc}, 2}^{p}$ by

$$
\begin{aligned}
\operatorname{var} G_{\mathrm{mt}(1)}^{[7(a)]}= & \frac{2 N_{L}^{2} N_{R}^{2}}{\left(N_{L}+N_{R}\right)^{4}} \frac{2 \tau_{D}}{\left(p_{2 k+3}+p_{2 k+1}-2 p_{2 k+2}\right)} \\
& \times \int_{-c}^{c} d \boldsymbol{s} d \boldsymbol{u} \frac{T_{H}^{2}}{\Omega^{2}} \frac{e^{(i / \hbar)} \frac{\boldsymbol{u} u}{t_{\mathrm{enc}, 1} t_{\mathrm{enc}, 2}}}{\int_{t_{\mathrm{encs}} /(2 k+1)}^{t_{\mathrm{enc}, \min } / k} d \tau_{p}} \\
& \times e^{-p_{2 k+1} \tau_{p} / \tau_{D}}\left[e^{-\left(p_{2 k+2}-p_{2 k+1}\right)\left(t_{\mathrm{encs}}-2 k \tau_{p}\right) / \tau_{D}}\right. \\
& -e^{-\left(p_{2 k+3}-p_{2 k+2}\right)\left(t_{\mathrm{enc}, \min }-k \tau_{p}\right) / \tau_{D}} \\
& \left.\times e^{-\left(p_{2 k+2}-p_{2 k+1}\right)\left(t_{\mathrm{enc}, \max }-k \tau_{p}\right) / \tau_{D}}\right],
\end{aligned}
$$

and second for $t_{\mathrm{enc}, \max }^{p}<\tau_{p}<t_{\mathrm{enc}, 1}^{p}+t_{\mathrm{enc}, 2}^{p}$ by

$$
\begin{aligned}
\operatorname{var} G_{\mathrm{mt}(2)}^{[7(a)]}= & \frac{2 N_{L}^{2} N_{R}^{2}}{\left(N_{L}+N_{R}\right)^{4}} \frac{2 \tau_{D}}{\left(p_{2 k+3}+p_{2 k+1}-2 p_{2 k+2}\right)} \\
& \times \int_{-c}^{c} d \boldsymbol{s} d \boldsymbol{u} \frac{T_{H}^{2}}{\Omega^{2}} \frac{e^{(i / \hbar) s \boldsymbol{u}}}{t_{\mathrm{enc}, 1} t_{\mathrm{enc}, 2}} \int_{t_{\mathrm{enc}, \max } /(k+1)}^{t_{\mathrm{encs}} /(2 k+1)} d \tau_{p} \\
& \times\left[e^{-\left(2 p_{2 k+2}-p_{2 k+3}\right) \tau_{p} / \tau_{D}}\right.
\end{aligned}
$$




$$
\begin{aligned}
& \times e^{-\left(p_{2 k+3}-p_{2 k+2}\right)\left(t_{\mathrm{encs}}-2 k \tau_{p}\right) / \tau_{D}} \\
& -e^{-\left(p_{2 k+3}-p_{2 k+2}\right)\left(t_{\mathrm{en}, \min }-k \tau_{p}\right) / \tau_{D}} \\
& \times e^{-\left(p_{2 k+2}-p_{2 k+1}\right)\left(t_{\mathrm{enc}, \max }-k \tau_{p}\right) / \tau_{D}} \\
& \left.\times e^{-p_{2 k+1} \tau_{p} / \tau_{D}}\right] .
\end{aligned}
$$

In the case of the two encounters overlapping at both ends the contribution is obtained to be

$$
\begin{aligned}
\operatorname{var} G_{\mathrm{mt}}^{[7(b)]}= & \frac{2 N_{L}^{2} N_{R}^{2}}{\left(N_{L}+N_{R}\right)^{4}} \int_{-c}^{c} d \boldsymbol{s} d \boldsymbol{u} \int_{t_{\mathrm{enc}, \max } /(k+1)}^{t_{\mathrm{encs}} /(2 k+1)} d \tau_{p} \frac{T_{H}^{2}}{\Omega^{2}} \\
& \times \frac{e^{(i / \hbar) s \boldsymbol{u}}}{t_{\mathrm{encs}}}\left[t_{\mathrm{encs}}-(2 k+1) \tau_{p}\right] \\
& \times e^{-\left(p_{2 k+3}-p_{2 k+2}\right)\left(t_{\mathrm{encs}}-2 k \tau_{p}\right) / \tau_{D}} e^{-\left(2 p_{2 k+2}-p_{2 k+3}\right) \tau_{p} / \tau_{D}} .
\end{aligned}
$$

\section{APPENDIX B: DIFFERENT TUNNELING PROBABILITIES}

Here we generalize Eq. (25) to the case of different $\Gamma_{j}$ for the different lead modes. Factors like $\Gamma N$ are then replaced by a sum over the $\Gamma_{j}$ with respect to the $N$ open channels. An analogous replacement is made for the $p_{j}$ for $j \geqslant 1$. In order to keep the notation compact we introduce

$$
G_{(i)} \equiv \sum_{j=1}^{N_{i}} \Gamma_{j}, \quad G_{i} \equiv \sum_{j=1}^{N_{L}+N_{R}} p_{j}
$$

For example, the contribution from the diagram in Fig. 4(a), previously given in Eq. (16), becomes

$$
\operatorname{var} G^{[4]}=\frac{G_{(1)}^{2} G_{(2)}^{2} G_{2}^{2}}{G_{1}^{6}},
$$

where the Ehrenfest-time dependence is the same as in Eq. (16) although we set $\tau_{E}=0$ here. The first two terms in the numerator result from the channel summations. The third term replaces the factor $p_{2}^{2} N^{2}$ in Eq. (16) and the denominator replaces the previous $(\Gamma N)^{4}$.

To keep the contributions from configurations where one link connecting the encounter to the opening is removed in a compact form, we define

$$
H_{(i), k} \equiv \sum_{j=1}^{N_{i}} \Gamma_{j}\left(1-\Gamma_{j}\right)^{k-1}
$$

Considering again the corresponding contribution from Fig. 4(a), previously given in Eq. (17), we have

$$
\operatorname{var} G^{[4-1 l]}=-\frac{2\left[H_{(1), 2} G_{(2)}+G_{(1)} H_{(2), 2}\right] G_{2} G_{(1)} G_{(2)}}{G_{1}^{5}} .
$$

The first term derives from when the link connecting the encounter to lead 1 is shrunk while the second term corresponds to when the link connecting an encounter to lead 2 is removed. Compared to Eq. (B2), the factor $H_{(i), k}$ takes into account that the particle is entering the system in a certain channel and returns to the same channel after traversing a link.
Removing two links from different encounters likewise leads to

$$
\operatorname{var} G^{[4(a)-2 l]}=\frac{4 G_{(1)} H_{(1), 2} H_{(2), 2} G_{(2)}}{G_{1}^{4}} .
$$

For the diagram in Fig. 4(a) we can also remove two links from the same encounter, and for this we define

$$
I_{(i), k} \equiv \sum_{j=1}^{N_{i}} \Gamma_{j}^{2}\left(1-\Gamma_{j}\right)^{k-1}
$$

so that we obtain

$$
\begin{aligned}
\operatorname{var} G^{[4(a)-2 l(s)]}=-\frac{\left[I_{(1), 1} G_{(2)}^{2}+G_{(1)}^{2} I_{(2), 1}\right] G_{2}}{G_{1}^{4}}, \\
\operatorname{var} G^{[4(a)-3 l]}=\frac{2\left[G_{(1)} H_{(1), 2} I_{(2), 1}+I_{(1), 1} H_{(2), 2} G_{(2)}\right]}{G_{1}^{3}}, \\
\\
\operatorname{var} G^{[4(a)-4 l]}=\frac{I_{(1), 1} I_{(2), 1}}{G_{1}^{2}} .
\end{aligned}
$$

For the diagram in Fig. 4(b), we cannot shrink two links attached to the same encounter (or more than two links) and because of the way the encounters are arranged when we shrink two links we obtain

$$
\begin{aligned}
\operatorname{var} G^{[4(b)-2 l]}= & \frac{2 G_{(1)} H_{(1), 2} H_{(2), 2} G_{(2)}}{G_{1}^{4}} \\
& +\frac{H_{(1), 2}^{2} G_{(2)}^{2}+G_{(1)}^{2} H_{(2), 2}^{2}}{G_{1}^{4}} .
\end{aligned}
$$

Using the definitions above, this is equal to Eq. (B5) plus the results from Eqs. (B7)-(B9) so that time-reversal symmetry still leads simply to a factor of 2 .

In an analogous manner, we can show that each diagram in Fig. 5 gives twice the contribution of the diagram in Fig. 4(b) while for the diagrams in Fig. 6 we obtain

$$
\begin{gathered}
\operatorname{var} G^{[6]}=-\frac{2 G_{(1)}^{2} G_{(2)}^{2} G_{3}}{G_{1}^{5}}, \\
\operatorname{var} G^{[6-1 l]}=\frac{4\left[G_{(1)} H_{(1), 3} G_{(2)}^{2}+G_{(1)}^{2} H_{(2), 3} G_{(2)}\right]}{G_{1}^{4}}, \\
\operatorname{var} G^{[6-2 l]}=\frac{2\left[I_{(1), 2} G_{(2)}^{2}+G_{(1)}^{2} I_{(2), 2}\right]}{G_{1}^{3}} .
\end{gathered}
$$

Summing all these contributions, we obtain the RMT result $^{28}$ for the leading order in $1 / N$ contribution to the conductance variance

$$
\begin{aligned}
\operatorname{var} G^{\mathrm{RMT}}= & \frac{1}{\left(g_{1}+g_{1}^{\prime}\right)^{6}}\left[2 g_{1}^{5} g_{2}^{\prime}-2 g_{1}^{4} g_{2}^{\prime} g_{1}^{\prime}-4 g_{1}^{3} g_{2}^{\prime} g_{1}^{\prime 2}\right. \\
& -4 g_{1}^{2} g_{2} g_{1}^{\prime 3}-2 g_{1} g_{2} g_{1}^{\prime 4}+2 g_{2} g_{1}^{\prime 5}+2 g_{1}^{4} g_{1}^{\prime 2} \\
& +4 g_{1}^{3} g_{1}^{\prime 3}+2 g_{1}^{2} g_{1}^{\prime 4}+3 g_{1}^{4} g_{2}^{\prime 2}+6 g_{1}^{2} g_{2} g_{2}^{\prime} g_{1}^{\prime 2} \\
& +3 g_{2}^{2} g_{1}^{\prime 4}-2 g_{1}^{5} g_{3}^{\prime}-2 g_{1}^{4} g_{3}^{\prime} g_{1}^{\prime}-4 g_{1} g_{3} g_{1}^{\prime 4} \\
& \left.-2 g_{3} g_{1}^{\prime 5}\right],
\end{aligned}
$$


for systems without time-reversal symmetry, and twice this result for those with. Here the notation introduced in Ref. 28

$$
g_{k}=\sum_{n=1}^{N_{L}} \Gamma_{n}^{k}, \quad g_{k}^{\prime}=\sum_{n=1}^{N_{R}} \Gamma_{n}^{k}
$$

was used.

\section{APPENDIX C: SHOT NOISE} $\operatorname{as}^{40}$

Here we calculate the shot-noise power. It can be written

$$
P=G-h=\left\langle\operatorname{Tr}\left[\mathbf{t t}^{\dagger}\right]\right\rangle-\left\langle\operatorname{Tr}\left[\left(\mathbf{t t}^{\dagger}\right)^{2}\right]\right\rangle .
$$

The average conductance $G(E)$ has previously been calculated, ${ }^{33,41}$ and the first few terms for systems with time-reversal symmetry can be written as

$$
\begin{aligned}
G(E)= & \frac{G_{(1)} G_{(2)}}{G_{1}}-\left(\frac{2}{\beta}-1\right) \frac{G_{(1)} G_{(2)} G_{2}}{G_{1}^{3}} \\
& +\left(\frac{2}{\beta}-1\right) \frac{G_{(1)} H_{(2), 2}+H_{(1), 2} G_{(2)}}{G_{1}^{2}}+\cdots,
\end{aligned}
$$

using the notation of Appendix B. The second two terms derive from the diagrams in Fig. 3 so that without timereversal symmetry only the first term in Eq. (C2) remains. This is included as $\beta=2$ for systems without time-reversal symmetry and $\beta=1$ for those with time-reversal symmetry. The function $h$ is given semiclassically in terms of four trajectories,

$$
\begin{aligned}
h= & \left\langle\frac{1}{T_{H}^{2}} \sum_{\substack{a, b \\
c, d}} \sum_{\substack{\gamma(a \rightarrow b) \\
\gamma^{\prime}(c \rightarrow b)}} \sum_{\substack{\xi(c \rightarrow d) \\
\xi^{\prime}(a \rightarrow d)}} A_{\gamma} A_{\gamma^{\prime}}^{*} A_{\xi} A_{\xi^{\prime}}^{*}\right. \\
& \left.\times e^{(i / \hbar)\left(S_{\gamma}-S_{\gamma^{\prime}}+S_{\xi}-S_{\xi^{\prime}}\right)}\right\rangle,
\end{aligned}
$$

where the main difference from the conductance variance (7) is that the trajectories connect different channels and the sum is unrestricted. We start with the diagram from Fig. 11, where since the partner trajectories cross over in the encounter they automatically travel from and to the correct channels and all four channels are unrestricted. This means that this structure is now lower order (in inverse channel number) than it was for the conductance variance (it was there contained by taking into account the diagram in Fig. 4(a) and removing two links connected to the same encounter). For the diagram in Fig. 11, we therefore have

$$
\begin{aligned}
h^{[11]}= & -\frac{G_{(1)}^{2} G_{(2)}^{2} G_{2}}{G_{1}^{4}} \\
& +\frac{2\left[G_{(1)} H_{(1), 2} G_{(2)}^{2}+G_{(1)}^{2} H_{(2), 2} G_{(2)}\right]}{G_{1}^{3}} \\
& +\frac{G_{(1)}^{2} I_{(2), 1}+I_{(1), 1} G_{(2)}^{2}}{G_{1}^{2}} .
\end{aligned}
$$

This contribution, along with its Ehrenfest-time dependence, was previously calculated in Ref. 33 .

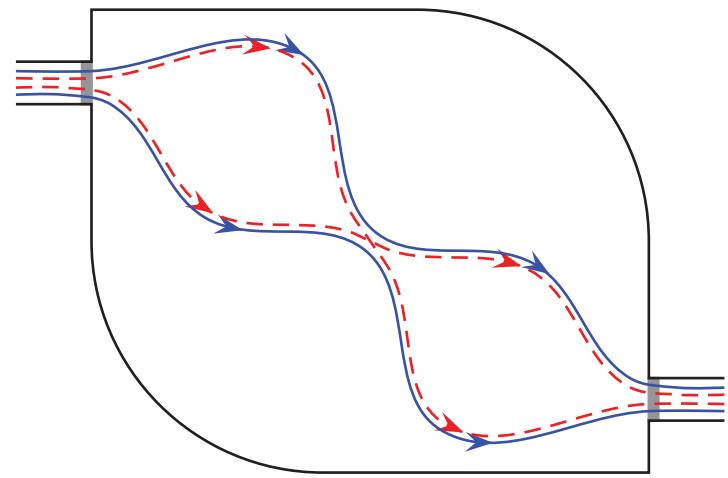

FIG. 11. (Color online) Two trajectories with a single encounter and two partner trajectories.

Moving to the next order term, which has recently been calculated using $\mathrm{RMT}^{42}{ }^{4}$ we again simply need to look at the corresponding structures and consider the possible ways of shrinking links. Without time-reversal symmetry there are no possible structures at this order, so we therefore consider systems with time-reversal symmetry. The structures that contribute are depicted in Fig. 2 of Ref. 15. There is also, in Fig. 2(d) there, a quadruplet involving two independent pairs, one of which is simply a diagonal pair, while the other involves a single two-encounter, as in Fig. 3 of this paper. Because the start (or end) channels must coincide with $a=c$ (or $b=d$ ) there is a subtlety when we consider shrinking one link. If we shrink the link on the left (or right) side of the two-encounter so that it moves into the lead where the diagonal pair emanates (or terminates) we actually have the same case as when the three-encounter from Fig. 2(c) (of Ref. 15) moves into the lead. As such, we include this case there [i.e. in the contribution from Fig. 2(c)], leaving for Fig. 2(d) a contribution of

$$
\begin{aligned}
h^{[2(\mathrm{~d})]}= & -\frac{2\left[G_{(1)}^{2} I_{(2), 1}+I_{(1), 1} G_{(2)}^{2}\right] G_{2}}{G_{1}^{4}} \\
& +\frac{2\left[G_{(1)} H_{(1), 2} I_{(2), 1}+I_{(1), 1} H_{(2), 2} G_{(2)}\right]}{G_{1}^{3}} .
\end{aligned}
$$

The diagram in Fig. 2(a) of Ref. 15 is similar to the diagram in Fig. 4(b) here and we obtain

$$
\begin{aligned}
h^{[2(\mathrm{a})]}= & \frac{4 G_{(1)}^{2} G_{(2)}^{2} G_{2}^{2}}{G_{1}^{6}}+\frac{8 G_{(1)} H_{(1), 2} H_{(2), 2} G_{(2)}}{G_{1}^{4}} \\
& -\frac{8\left[G_{(1)} H_{(1), 2} G_{(2)}^{2}+G_{(1)}^{2} H_{(2), 2} G_{(2)}\right] G_{2}}{G_{1}^{5}} \\
& +\frac{2\left[G_{(1)}^{2} H_{(2), 2}^{2}+H_{(1), 2}^{2} G_{(2)}^{2}\right]}{G_{1}^{4}} .
\end{aligned}
$$

For the diagram in Fig. 2(b) of Ref. 15 we obtain twice the result of Fig. 5(a) here [or four times the result of Fig. 4(b)] for the conductance variance. For the diagram in Fig. 2(c) of Ref. 15 we likewise obtain twice the result of Fig. 6(a) here for the conductance variance. With all the next-to-leading-order shot-noise contributions, we can combine them and indeed find the same result as in Ref. 42. 
We can simplify the result by setting all of the tunneling probabilities equal to $\Gamma$ :

$$
\begin{aligned}
P(\Gamma)= & \frac{\Gamma(1-\Gamma) N_{1} N_{2}}{\left(N_{L}+N_{R}\right)}+\frac{\Gamma(3 \Gamma-2) N_{1}^{2} N_{2}^{2}}{\left(N_{L}+N_{R}\right)^{3}} \\
& +\left(\frac{2}{\beta}-1\right)\left(\frac{\Gamma(4 \Gamma-3) N_{1} N_{2}\left(N_{1}-N_{2}\right)^{2}}{\left(N_{L}+N_{R}\right)^{4}}\right) \\
& +\cdots
\end{aligned}
$$

Setting $\Gamma=1$, we also recreate the first two terms,

$$
\begin{aligned}
P(\Gamma=1)= & \frac{N_{1}^{2} N_{2}^{2}}{\left(N_{L}+N_{R}\right)^{3}} \\
& +\left(\frac{2}{\beta}-1\right)\left(\frac{N_{1} N_{2}\left(N_{1}-N_{2}\right)^{2}}{\left(N_{L}+N_{R}\right)^{4}}\right)+\cdots,
\end{aligned}
$$

of the result in Ref. 15 .
${ }^{1}$ P. Ehrenfest, Z. Phys. 45, 455 (1927).

${ }^{2}$ B. V. Chirikov, F. M. Izrailev, and D. L. Shepelyansky, Sov. Sci. Rev. Sect. C 2, 209 (1981)

${ }^{3}$ I. L. Aleiner and A. I. Larkin, Phys. Rev. B 54, 14423 (1996).

${ }^{4}$ O. Yevtushenko, G. Lütjering, D. Weiss, and K. Richter, Phys. Rev. Lett. 84, 542 (2000).

${ }^{5}$ İ. Adagideli, Phys. Rev. B 68, 233308 (2003).

${ }^{6}$ P. W. Brouwer and S. Rahav, Phys. Rev. B 74, 075322 (2006).

${ }^{7}$ R. S. Whitney and Ph. Jacquod, Phys. Rev. Lett. 94, 116801 (2005); Ph. Jacquod and R. S. Whitney, Phys. Rev. B 73, 195115 (2006).

${ }^{8}$ D. Waltner and J. Kuipers, Phys. Rev. E 82, 066205 (2010).

${ }^{9} \mathrm{Ph}$. Jacquod and E. V. Sukhorukov, Phys. Rev. Lett. 92, 116801 (2004).

${ }^{10}$ J. Tworzydło, A. Tajic, H. Schomerus, P. W. Brouwer, and C. W. J. Beenakker, Phys. Rev. Lett. 93, 186806 (2004).

${ }^{11}$ A. Altland, P. W. Brouwer, and C. Tian, Phys. Rev. Lett. 99, 036804 (2007).

${ }^{12}$ C. Petitjean, P. Jacquod, and R. S. Whitney, JETP Lett. 86, 647 (2007); R. S. Whitney, Ph. Jacquod, and C. Petitjean, Phys. Rev. B 77, 045315 (2008).

${ }^{13}$ O. Agam, I. L. Aleiner, and A. I. Larkin, Phys. Rev. Lett. 85, 3153 (2000).

${ }^{14}$ R. S. Whitney and Ph. Jacquod, Phys. Rev. Lett. 96, 206804 (2006).

${ }^{15}$ P. Braun, S. Heusler, S. Müller, and F. Haake, J. Phys. A 39, L159 (2006).

${ }^{16}$ G. Berkolaiko, J. M. Harrison, and M. Novaes, J. Phys. A 41, 365102 (2008).

${ }^{17}$ D. Waltner, J. Kuipers, and K. Richter, Phys. Rev. B 83, 195315 (2011).

${ }^{18}$ I. L. Aleiner and A. I. Larkin, Phys. Rev. E 55, R1243 (1997).

${ }^{19}$ C. Tian and A. I. Larkin, Phys. Rev. B 70, 035305 (2004).

${ }^{20}$ P. W. Brouwer, S. Rahav, and C. Tian, Phys. Rev. E 74, 066208 (2006).

${ }^{21}$ D. Waltner, M. Gutiérrez, A. Goussev, and K. Richter, Phys. Rev. Lett. 101, 174101 (2008).
${ }^{22}$ M. Gutiérrez, D. Waltner, J. Kuipers, and K. Richter, Phys. Rev. E 79, 046212 (2009).

${ }^{23}$ B. Gutkin, D. Waltner, M. Gutiérrez, J. Kuipers, and K. Richter, Phys. Rev. E 81, 036222 (2010).

${ }^{24}$ H. U. Baranger, R. A. Jalabert, and A. D. Stone, Chaos 3, 665 (1993).

${ }^{25}$ K. Richter and M. Sieber, Phys. Rev. Lett. 89, 206801 (2002).

${ }^{26}$ K. Richter, Semiclassical Theory of Mesoscopic Quantum Systems (Springer, Berlin, Heidelberg, 2000).

${ }^{27}$ P. W. Brouwer and S. Rahav, Phys. Rev. B 74, 085313 (2006).

${ }^{28}$ P. W. Brouwer and C. W. J. Beenakker, J. Math. Phys. 37, 4904 (1996).

${ }^{29}$ M. Büttiker, Phys. Rev. Lett. 57, 1761 (1986).

${ }^{30}$ Y. Imry, in Directions in Condensed Matter Physics, edited by G. Grinstein and G. Mazenko (World Scientific, Singapore, 1986), p. 101.

${ }^{31}$ D. S. Fisher and P. A. Lee, Phys. Rev. B 23, 6851 (1981).

${ }^{32}$ S. Heusler, S. Müller, P. Braun, and F. Haake, Phys. Rev. Lett. 96, 066804 (2006); S. Müller, S. Heusler, P. Braun, and F. Haake, New J. Phys. 9, 1 (2007).

${ }^{33}$ R. S. Whitney, Phys. Rev. B 75, 235404 (2007).

${ }^{34}$ S. Müller, S. Heusler, P. Braun, F. Haake, and A. Altland, Phys. Rev. Lett. 93, 014103 (2004); Phys. Rev. E 72, 046207 (2005).

${ }^{35}$ F. M. Izrailev, Phys. Rep. 196, 299 (1990).

${ }^{36}$ Y. V. Fyodorov and H.-J. Sommers, JETP Lett. 72, 422 (2000); R. O. Vallejos and A. M. Ozorio de Almeida, Ann. Phys. 278, 86 (1999).

${ }^{37}$ J. Tworzydło, A. Tajic, H. Schomerus, and C. W. J. Beenakker, Phys. Rev. B 68, 115313 (2003).

${ }^{38}$ H. Schomerus and Ph. Jacquod, J. Phys. A 38, 10663 (2005).

${ }^{39}$ C. Tian, A. Kamenev, and A. Larkin, Phys. Rev. Lett. 93, 124101 (2004); Phys. Rev. B 72, 045108 (2005).

${ }^{40}$ M. Büttiker, Phys. Rev. Lett. 65, 2901 (1990).

${ }^{41}$ J. Kuipers, J. Phys. A 42, 425101 (2009).

${ }^{42}$ J. G. G. S. Ramos, A. L. R. Barbosa, and A. M. S. Macêdo, Phys. Rev. B 78, 235305 (2008). 